\title{
Ejercicio terapéutico para epicondilalgia lateral: revisión sistemática
}

\author{
F. Araya Quintanilla1 y V. Moyano Galvez ${ }^{2}$ \\ ${ }^{1}$ Kinesiología. ${ }^{2}$ Enfermería. Escuela de Enfermería. Universidad Católica de Chile. Chile
}

Araya Quintanilla F, Moyano Galvez V. Ejercicio terapéutico para epicondilalgia lateral: revisión sistemática. Rev Soc Esp Dolor 2015;22(6):253-270.

\begin{abstract}
The lateral epicondylalgia is one of the most common injuries of the arm; with the muscle extensor carpi radialis brevis (ERCB) affected the structure, repetitive motion, rapids and monotonous, mainly affects between $1-3 \%$ of the general population, being more common in women. Therapeutic exercise is one of the key elements in programs designed for the management of this condition.

There will be a synthesis of evidence through a systematic review of randomized clinical trials that established the effectiveness of exercise therapy in the management of these patients. The aim of this review is to determine whether there is scientific evidence to support the clinical effectiveness of exercise therapy alone or in a treatment program for adult patients with LE.

The search strategy included randomized clinical trials (RCTs) and controlled clinical trials (CCTs) databases were used: Medline, CINAHL, PEDro, Central, Lilacs. The results obtained were thirty-six articles that met our eligibility criteria. We conclude that there is moderate evidence shows that therapeutic exercise alone compared with a single intervention, decreases pain, improves function and increases strength pain free in short, medium and long term. There is strong evidence that exercise therapy reduces pain, increases strength and free from short-term pain. There is strong evidence that exercise therapy reduces pain, increases strength and improves functionality in the short, medium and long term.
\end{abstract}

Key words: Tennis elbow. Lateral epicondilytis. Exercise therapy. Home based exercise. Randomized clinical trial.

\section{RESUMEN}

La epicondilalgia lateral es una de las lesiones más comunes del brazo, siendo el músculo extensor radial corto del carpo (ERCB) la estructura más afectada, por movimientos repetitivos, rápidos y monótonos, principalmente afecta entre 1-3\% de la población general, siendo más común en mujeres. El ejercicio terapéutico, es uno de los elementos claves en los programas diseñados para el manejo de esta condición. Se realizará una búsqueda de la evidencia a través de una revisión sistemática de ensayos clínicos aleatorizados que hayan determinado la efectividad del ejercicio terapéutico para el manejo de estos pacientes. El objetivo de esta Revisión es determinar si existe evidencia científica que avale la efectividad clínica del ejercicio terapéutico sólo o dentro de un programa de tratamiento en pacientes adultos con epicondilalgia lateral.

La estrategia de búsqueda incluyó ensayos clínicos aleatorizados (ECA) y ensayos clínicos controlados (ECC); las bases de datos usadas fueron: Medline, Cinahl, PEDro, Central, Lilacs. Los resultados obtenidos fueron de treinta y seis artículos que cumplían con nuestros criterios de elegibilidad. Se concluye que existe evidencia moderada que el ejercicio terapéutico comparado con una intervención única, disminuye el dolor, mejora la funcionalidad y aumenta la fuerza libre de dolor en corto, medio y largo plazo. Existe evidencia de que el ejercicio terapéutico disminuye el dolor, y aumenta la fuerza sin dolor a corto plazo. Existe evidencia fuerte de que el ejercicio terapéutico disminuye el dolor, aumenta la fuerza y mejora la funcionalidad a corto, medio y largo plazo.

Palabras clave: Codo de tenista. Epicondilitis lateral. Ejercicio terapéutico. Programa de ejercicios en casa. Ensayo clínico aleatorizado. 


\section{INTRODUCCIÓN}

El término "tendinopatía" es usado preferentemente para describir varias patologías del tendón, incluyendo paratendinitis, tendinitis y tendinosis (1). La tendinopatía lateral del codo comúnmente referida como epicondilitis lateral (EL), es una de las lesiones más comunes del brazo, siendo el músculo extensor radial corto del carpo (ERCB) la estructura más afectada (2). Se define como un síndrome de dolor en el área del epicóndilo lateral (3-5), causado por el exceso de movimientos repetitivos, rápidos y monótonos (6-9), teniendo como principales síntomas el dolor, la disminución de la función de agarre y resistencia en la flexión dorsal de muñeca (10-12). Esta condición músculo esquelética afecta principalmente entre 1-3\% de la población general (13-23), incrementándose a un $15 \%$ en trabajadores de industrias que requieren tareas manuales repetitivas (24). Además, aunque ocurre en todas las edades (25), afecta predominantemente entre los 30 y 60 años $(2,26,27)$. A pesar que la proporción de los pacientes afectados por esta patología no está influenciado por el sexo del paciente, parece ser que la mayor duración y gravedad de la injuria se encuentra en las mujeres (4). Aun así la duración promedio de los síntomas es muy variable; se reportan rangos entre 6 meses a 2 años en pacientes que no han recibido tratamiento (28). Gran parte de los mecanismos patogénicos de las tendinopatías no están claramente establecidos (29); aún siguen siendo un tema de debate en la literatura; si estas corresponden a un proceso inflamatorio o degenerativo. A pesar de que datos histopatológicos son categóricos en concluir que la tendinopatía no es una condición de tipo inflamatoria (30). Sino que más bien, sugieren que corresponde a la presencia de colágeno desorganizado más que a la presencia de células inflamatorias (31-33). Por otra parte resulta importante mencionar que cuando el tendón está sobrecargado o sometido a estos microtraumatismos repetitivos, se traducen en la desorganización de las fibras de colágeno, aumento de fibroblastos, presencia de proteoglicanos y glicosaminoglicanos (10,34). Por lo tanto, dichas injurias acumulativas no sólo debilitaría al colágeno sino que además se asocian a cambios patológicos de la matriz extracelular (MEC) y a elementos vasculares del tendón $(35,36)$. Todo esto debiera ser considerado en un análisis para las distintas intervenciones recomendadas para el tratamiento de esta condición músculo esquelética. Numerosos tratamientos han sido descritos en la literatura para las tendinopatías dependiendo de su fase de presentación $(37,38)$, siendo las inyecciones de corticoides y la fisioterapia los tratamientos más comunes $(39,40)$. Es por esto, que como en una primera instancia se establece la reducción de la sintomatología a través de hielo, reposo relativo (41) y algunas modalidades fisioterapéuticas como ultrasonido y láser (42). Es necesario establecer que existe una evidencia insuficiente con respecto a su efectividad a largo plazo (43) Pero cabe destacar, que por más que existan tratamientos con diferentes mecanismos teóricos de acción, todos tienen un mismo objetivo, en reducir el dolor y mejorar la función (44). Sin embargo; estudios han señalado que a pesar de la resolución de síntomas mediante tratamientos conservadores permanece el déficit sensoriomotor (45), cuyas alteraciones se encuentran presentes de forma bilateral en pacientes con EL $(46,47)$. Cabe destacar que el sistema sensoriomotor se define como un componente del control motor, el cual se utiliza para describir su componente sensorial y la integración del procesamiento central junto con los elementos implicados en el mantenimiento de la estabilidad durante el movimiento dinámico (48), que incluye la posición sensorial de la articulación, sensación de fuerza y control neuromuscular, implicando ambos mecanismos de feedback y feedforward (49). A su vez, se ha establecido que el tiempo de reacción y la velocidad del movimiento son medidas simples de la función sensoriomotora, esto se ha demostrado que en pacientes con EL resulta ser significativamente más lento comparado con pacientes sanos $(46,47)$. Este déficit ha sido identificado previamente en otras condiciones músculo esqueléticas crónicas tales como el síndrome de dolor patelofemoral (50), síndrome de dolor lumbar $(51,52)$ y trastornos de hombro $(53,54)$. Por lo general, el tratamiento conservador está dirigido en disminuir el dolor y mejorar la funcionalidad en pacientes con EL. Algunos autores $(23,55,56)$ sugieren que la mayoría de estos pacientes reportan una mejoría en su condición a largo plazo, en particular, si adoptan un programa de terapia física y ejercicio terapéutico. Siendo ser más eficaz que la inyección de corticoides $(46,47)$. Cabe destacar; que un buen diseño de ejercicio terapéutico individual es siempre un componente fundamental en la terapia física, cuyo objetivo resulta ser el logro de un nivel óptimo de movimiento básico hasta llegar a niveles de actividad física complejas, a su vez, tener presente el impacto que tiene este sobre los efectos en los tejidos. El ejercicio terapéutico toma su lugar, como uno de los elementos claves en los programas diseñados para mejorar o restaurar la función (54). Y resulta sistemático, por el desempeño de los movimientos corporales, posturas físicas, o actividades destinadas a proporcionar al paciente el medio para prevenir el deterioro como así también; restaurar o mejorar la función física, optimizando así el estado general de salud o sentido de bienestar. Las técnicas seleccionadas para un ejercicio terapéutico individualizado se basan en la determinación de las causas subyacentes de las deficiencias o limitaciones funcionales de un paciente (57).

Es fundamental destacar el rol del ejercicio ya que sigue siendo unos de los tratamientos más comunes (58-60), como así también, ha demostrado tener efectos positivos en esta patología (61). De acuerdo a esto; la literatura sugiere que un programa de ejercicio tanto de fortalecimiento como de estiramiento son componentes principales para el 
manejo de esta condición, ya que el tendón no sólo debe ser fuerte, sino que también flexible, estimulando de cierta manera los mecanoreceptores de los tenocitos, generando la síntesis de colágeno (60-65). Además de que el flujo sanguíneo se detiene en el área de daño, produciéndose neovascularización, lo cual mejora el flujo y reparación a largo plazo (66). Otro fundamento que se atribuye al ejercicio es la analgesia que provocaría, con mecanismos profundos en los tejidos después del ejercicio, involucrando los procesos moduladores periféricos y centrales del dolor (67-71). A su vez, se han recomendado programas de ejercicio excéntrico dentro de los cuales se incorpora la carga, la velocidad y la frecuencia de contracción como elementos relevantes al momento de realizar el ejercicio (72), como así también se plantean protocolos excéntricos isocinéticos desarrollados específicamente para el trabajo de esta condición pero del cual aún no ha sido rigurosamente investigado (73). No obstante; un método de tratamiento que está abarcando la atención es el fortalecimiento excéntrico (74). Puesto que este programa se basa en fortalecer tanto músculos y tendones del antebrazo (75). Es por esto, que algunos terapeutas confieren que la carga en los ejercicios excéntricos debe ser incrementada en función a los síntomas del paciente, pues de lo contrario existe una posibilidad de una reagudización $(76,77)$. Otro principio básico del ejercicio excéntrico es la velocidad, si bien es cierto, ha sido controversial puesto que algunos afirman que la velocidad debe ser incrementada en cada sesión de tratamiento lo que aumenta la carga del tendón (78-80), sin embargo también se ha señalado que la velocidad debe ser lenta para evitar una posible lesión (60). De lo mencionado anteriormente uno de los fundamentos para que el ejercicio sea a baja velocidad es probablemente el hecho que no exceda el límite elástico del tendón y genere así menos calor perjudicial para el tejido (76). El tercer principio básico de los ejercicios excéntricos es la frecuencia de contracciones. Las series y repeticiones pueden variar en la literatura, pero algunos autores han planteado tres series de diez repeticiones con el codo en extensión completa y el antebrazo en pronación puede ser beneficioso, ya que se plantea que se consigue el mejor fortalecimiento de los tendones, según la tolerancia del paciente $(64,76,81)$. Otro factor común en los programas de ejercicios excéntrico es que pueden ser desarrollados en el hogar sin necesidad de supervisión de visitas de manera regular (82-84).

Por otra parte los ejercicios de stretching son propuestos como eficaces para aumentar la flexibilidad en esta condición, entendiendo este concepto como el rango de movimiento posible por medio de una o más articulaciones $(84,86)$. Si bien es cierto; existe una variedad de técnicas para la flexibilidad tales como; balística, estática y facilitación neuromuscular propioceptiva, sin embargo no es una preocupación en cuanto a las técnicas o procedimientos que deben ser utilizados para los óptimos beneficios, aún así es la técnica estática la que resulta más utilizada $(78,79,87)$. El stretching estático está definido como un estiramiento pasivo dado en la unidad músculo-tendinosa, que se realiza en estiramiento máximo y mantenido en un período de tiempo $(64,79)$. En el caso de la EL, esta técnica debería ser realizada en el músculo ECRB, cuya posición se logra en extensión de codo, antebrazo en pronación, la muñeca en flexión y con desviación ulnar (64), con una duración de 3 a 60 segundos (80). Aunque algunos autores sugieren que desde 30 a 45 segundos es más efectivo el incremento de flexibilidad del tendón (87-90). El rol que cumple el programa de ejercicios en casa, resulta también fundamental en donde la seguridad del paciente mejora y el riesgo de lesión o recidiva se reduce al mínimo, para ello es importante la instrucción y educación de los pacientes. Es por esto, que se debe incorporar principios adecuados de la mecánica corporal y la protección de las articulaciones de manera de minimizar el riesgo de lesión (57).

De lo anteriormente expuesto nace nuestra pregunta de investigación: en la rehabilitación de pacientes esqueléticamente maduros con epicondilalgia lateral, ¿Existe evidencia que el ejercicio terapéutico sólo o dentro de un programa de tratamiento en comparación con otras intervenciones fisioterapéuticas es más efectivo en el manejo clínico de la epicondilalgia lateral?

\section{OBJETIVO}

Determinar si existe evidencia científica que avale la efectividad clínica del ejercicio terapéutico sólo o dentro de un programa de tratamiento en pacientes adultos con epicondilalgia lateral.

\section{Objetivo específico}

1. Determinar si existe evidencia científica que avale que el ejercicio sólo comparado con cualquier otra modalidad fisioterapéutica es más efectivo en disminuir el dolor, aumentar la fuerza y mejorar la funcionalidad en pacientes adultos con EL.

2. Determinar si existe evidencia científica que avale que el ejercicio dentro de un programa de tratamiento comparado con cualquier otra modalidad fisioterapéutica es más efectivo en disminuir el dolor, aumentar la fuerza y mejorar la funcionalidad en pacientes adultos con EL.

3. Determinar si existe evidencia científica que avale que el ejercicio dentro de un programa de tratamiento comparado con otro programa de tratamiento es más efectivo en disminuir el dolor, aumentar la fuerza y mejorar la funcionalidad en pacientes adultos con EL. 


\section{METODOLOGÍA}

\section{Tipos de estudios}

Para la realización de la presente Revisión Sistemática se desarrolló una estrategia de búsqueda en la cual se incluyeron Ensayos clínicos Aleatorizados (ECAs) y Ensayos clínicos Controlados (ECC) que cumplieran con nuestros criterios de elegibilidad.

\section{Bases de datos}

Se realizó una búsqueda electrónica en las siguientes bases de datos: MEDLINE, (www.ncbi.nlm.nih.gov/pubmed acceso el 03 de octubre del 2014) LILACS (www. bases.bireme acceso el 18 octubre del 2014), CINAHL (www.ebscohost.com/cinahl acceso 18 de octubre 2014), PEDro (www.pedro.org.au acceso el 10 de octubre del 2014) CENTRAL (www.cochrane.org acceso el 10 de octubre del 2014; se seleccionaron artículos publicados entre 03 de octubre del 1980 hasta 18 de octubre del 2014.

\section{Términos de búsqueda}

Los términos de búsqueda de nuestra revisión fueron obtenidos del MeSH (tesauro de PubMed) siendo algunos de ellos: exercise therapy, tennis elbow, physical therapy modalities. También se incluyó un término de texto libre: Lateral epicondylalgia. Para llevar a cabo la búsqueda en la base de datos MEDLINE se utilizó la estrategia de búsqueda sensible propuesta en el "Handbook Cochrane" (91) (Fig. 1).

Para las bases de datos Central, Cinahl, Lilacs y PEDro se realizará la estrategia de búsqueda con la combinación de los términos MeSH mencionados previamente.

\section{Límites de búsqueda}

\begin{tabular}{|l|l|}
\hline $\begin{array}{l}\text { Ensayos clínicos } \\
\text { aleatorizados y controlados }\end{array}$ & $\begin{array}{l}\text { Sin distinción de género } \\
\text { y raza }\end{array}$ \\
\hline $\begin{array}{l}\text { Pacientes con EL con } \\
\text { diagnóstico clínico y/o } \\
\text { imagenológico }\end{array}$ & $\begin{array}{l}\text { Artículos publicados en } \\
\text { idioma inglés o español }\end{array}$ \\
\hline $\begin{array}{l}\text { Pacientes mayores de 18 } \\
\text { años }\end{array}$ & $\begin{array}{l}\text { Artículos publicados } \\
\text { desde el 16 de octubre } \\
\text { 1980 hasta el 16 de } \\
\text { octubre de 2014 }\end{array}$ \\
\hline
\end{tabular}

\section{Criterios de selección}

A los artículos seleccionados por la búsqueda preliminar, se les aplicó una lectura crítica a los resúmenes y/o textos completos, estos fueron evaluados según los siguientes criterios:

\begin{tabular}{|l|l|}
\hline 1. Tennis elbow & 16. Concentric exercise \\
\hline 2. Lateral epicondylitis & 17. Eccentric exercise \\
\hline 3. Lateral epicondylalgia & \begin{tabular}{l} 
18. Manual therapy \\
\hline 4. Lateral epicondylosis
\end{tabular} \\
\hline 5. Lateral elbow tendinopathy & $\begin{array}{l}\text { 19. Home based exercise } \\
\text { \#16 OR \#17 OR \#18 OR \#19 }\end{array}$ \\
\hline 6. Lateral humeral epicondylalgia & \#9 AND \#20 \\
\hline 7. Lateral elbow pain & 22. Randomized clinical trial \\
\hline 8. Lateral humeral epicondylitis & 23. Randomized controlled trial \\
\hline 9. \# 1 OR \#2 OR \#3 OR \#4 OR \#5 OR \#6 OR \#7 OR \#8 & 24. Randomized \\
\hline 10. Physical therapy modalities & 25. \#22 OR \#23 OR \#24 \\
\hline 11. Exercise therapy & 26. Humans \\
\hline 12. Exercise & 27. Animals \\
\hline 13. Muscle stretching exercise & 28. \#26 NOT \#27 \\
\hline 14. Resistance training & 29. \#21 AND \#25 AND \#28 \\
\hline 15. Exercise programme & \\
\hline
\end{tabular}




\section{Criterios de inclusión}

- Artículos que hayan estudiado los beneficios del ejercicio terapéutico sólo o dentro de un programa de tratamiento.

- Artículos que hayan investigado tal tipo de ejercicios: Ejercicios concéntricos, ejercicios excéntricos, programa de ejercicios en casa, ejercicios de resistencia, ejercicios isocinéticos, ejercicios de stretching, ejercicios sensorios motrices.

- Artículos que hayan estudiado la efectividad clínica con métodos uni o multidimensionales para valorar dolor (EVA, escala numérica, escala de Mcgill, etc), fuerza de agarre (dinamometría), ROM (Goniometría), umbral doloroso, funcionalidad (DASH, PRTEEQ, PRFEQ).

\section{Criterios de exclusión}

- Artículos en fase preliminar, sin conclusión o interpretación de los resultados.

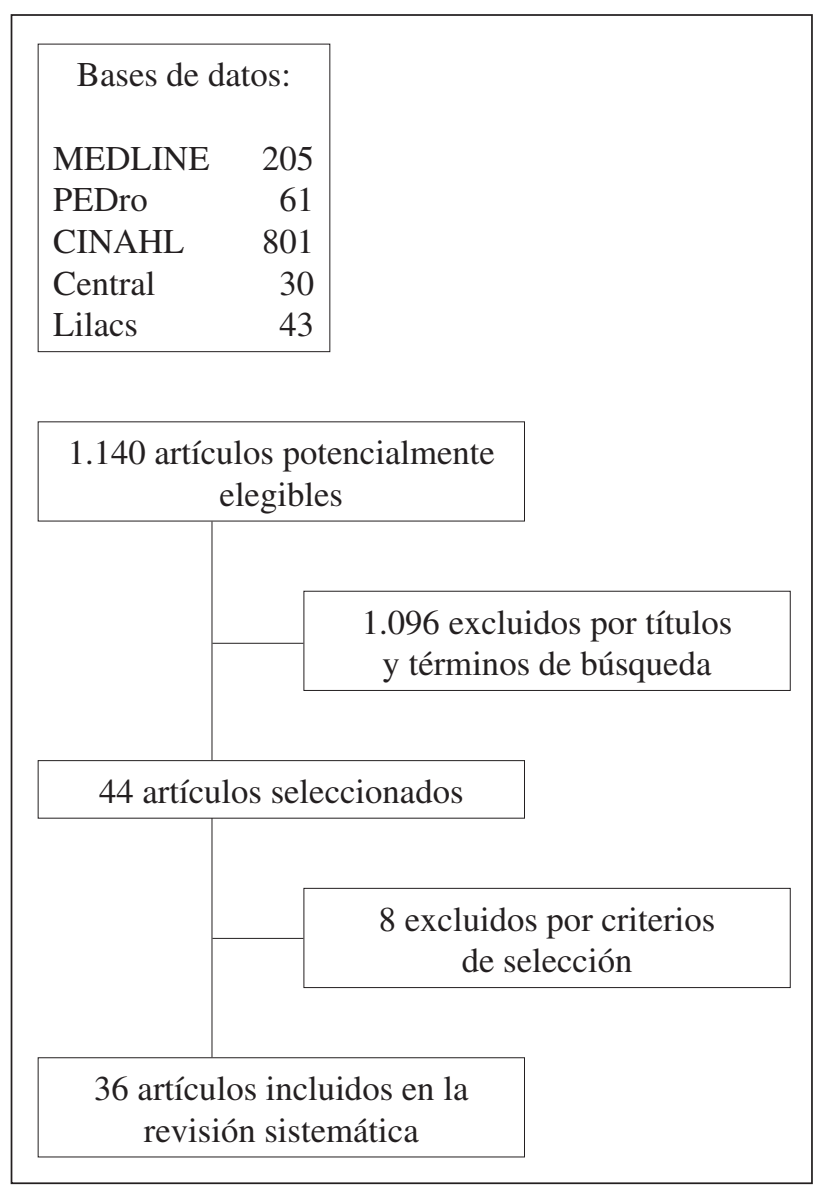

Fig. 1. Algoritmo de búsqueda.
- Artículos que presenten pacientes con EL experimental.

- Artículos con diseño metodológico crossover.

- Artículos de costo-efectividad.

- Artículos que evalúen parámetros cinéticos o cinemáticos.

\section{Evaluación del riesgo de sesgo de los artículos seleccionados}

Sobre la base de los criterios de selección de nuestra RS, sólo 36 artículos fueron incluidos (7-25,27,29,31,33-34,3740,43-45,61,73-75,82-84). El riesgo de sesgo se evaluará utilizando la herramienta propuesta en el "Cochrane Handbook” para Ensayos Clínicos Aleatorizados (91). Cada estudio será evaluado cualitativamente y los resultados serán presentados en una tabla especificando si cumple el criterio será representado con el color verde (bajo riesgo de sesgo), si no lo cumple con color rojo (alto riesgo de sesgo) y no claro con color amarillo (falta de información o incertidumbre sobre la potencial presencia de sesgo) (Tabla I).

Los 6 criterios a evaluar son:

- Generación de la secuencia de aleatorización.

- Ocultamiento de la secuencia de aleatorización.

- Cegamiento.

- Manejo adecuado de las pérdidas (análisis por intención de tratar).

- Reporte selectivo de resultados.

- Otros sesgos (sesgo publicación).

\section{Recopilación de datos}

Los dos autores (FA, VM) realizaron la cadena de búsqueda en forma independiente y seleccionaron los artículos que eran potencialmente elegibles, un asesores independientes (AI) evaluaron la calidad metodológica de los artículos seleccionados, en caso de desacuerdo y/o discrepancia los autores acordaron incorporar el estudio y en el análisis posterior decidir mediante discusión y consenso su inclusión final.

\section{Síntesis y análisis de datos}

En base al análisis de los datos extraídos de los artículos que cumplían los criterios de elegibilidad de nuestra revisión sistemática, al determinar la efectividad del ejercicio terapéutico en EL, debido a la heterogeneidad por la naturaleza de la intervención, no se pudo realizar la agrupación de datos en las diferentes medidas de resultados. Debido a la dispersión de tal intervención en cuanto a tipo y dosis es demasiado variable en cada estudio, por lo tanto, los resul- 
TABLA I. RIESGO DE SESGO DE LOS ARTÍCULOS INCLUIDOS

\begin{tabular}{|c|c|c|c|c|c|c|}
\hline & 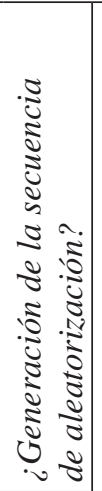 & 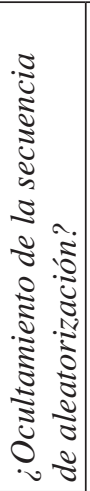 & 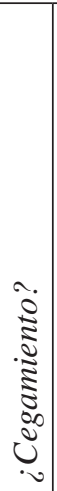 & 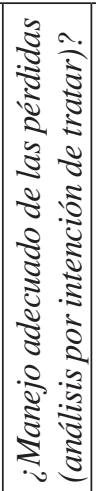 & 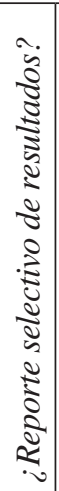 & 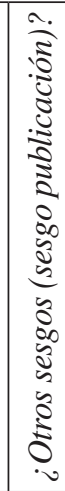 \\
\hline Ajimsha 2012 & $?$ & $?$ & + & - & + & + \\
\hline Bhardwaj 2011 & + & $?$ & $?$ & - & + & + \\
\hline Bisset 2006 & + & $?$ & + & + & + & + \\
\hline Bisset 2009 & $?$ & $?$ & + & + & + & + \\
\hline Blanchette 2011 & + & + & - & + & + & + \\
\hline Chung 2004 & + & + & + & + & + & + \\
\hline Coff 2009 & $?$ & - & $?$ & + & + & + \\
\hline Croisier 2007 & - & $?$ & + & - & + & + \\
\hline Dreschler 1997 & $?$ & $?$ & $?$ & + & + & + \\
\hline Hernández 2006 & + & $?$ & + & - & + & + \\
\hline Ho 2007 & - & $?$ & $?$ & + & + & + \\
\hline Kochar 2002 & + & $?$ & $?$ & + & + & + \\
\hline Luginbuhl 2008 & $?$ & $?$ & $?$ & - & + & + \\
\hline Manias 2006 & - & $?$ & $?$ & + & + & + \\
\hline Martinez-Silvestrini 2005 & - & $?$ & - & + & + & + \\
\hline Nagrale 2009 & $?$ & $?$ & + & + & + & + \\
\hline Newcomer 2001 & $?$ & $?$ & $?$ & - & + & + \\
\hline Nilsson 2007 & $?$ & $?$ & $?$ & - & + & + \\
\hline Oken 2008 & $?$ & $?$ & + & - & + & + \\
\hline Park 2010 & + & $?$ & $?$ & - & + & + \\
\hline Peterson 2011 & + & $?$ & - & + & + & + \\
\hline Pienimaki 1996 & $?$ & $?$ & + & + & + & + \\
\hline Pienimaki 1998 & $?$ & $?$ & $?$ & - & + & + \\
\hline Radpasand 2009 & + & + & + & + & + & + \\
\hline Smidt 2002 & + & + & + & + & + & + \\
\hline Stasinopoulos 2006 & - & $?$ & + & + & + & + \\
\hline Stasinopoulos 2009 & - & $?$ & + & - & + & + \\
\hline Stasinopoulos 2010 & - & $?$ & + & + & + & + \\
\hline Stergioulas 2007 & $?$ & + & + & + & + & + \\
\hline Struijs 2003 & $?$ & + & + & - & + & + \\
\hline Struijs 2004 & $?$ & $?$ & + & + & + & + \\
\hline Svemlöv 2001 & $?$ & $?$ & $?$ & - & + & + \\
\hline Söderberg 2011 & + & $?$ & $?$ & - & + & + \\
\hline Tyler 2010 & $?$ & $?$ & + & + & + & + \\
\hline Verhaar 1996 & $?$ & + & $?$ & + & + & + \\
\hline Viswas 2011 & $?$ & $?$ & + & + & + & + \\
\hline
\end{tabular}

tados de los artículos no se pueden combinar para llegar a un estimador puntual, debido a la disparidad en la homogeneidad clínica, que se considera cuando los pacientes, intervenciones, medidas de resultados y seguimiento son similares. Sin embargo, para llevar a cabo un meta-análisis se debe evaluar además la homogeneidad estadística, que es menos plausible de realizar debido que no se cumple el primer criterio.

\section{Criterios para valoración de los resultados}

De los 36 artículos seleccionados por nuestra búsqueda, no estaban en condiciones de poder agruparse en una comparación en base a un estimador puntual. Por este motivo, se utilizará un método cualitativo recomendado por el Grupo Cochrane de Espalda (91) con el uso de niveles de evidencia para la síntesis de los datos (Tabla II).

\section{RESULTADOS}

\section{Selección y características de los estudios}

De acuerdo a los criterios de nuestra revisión, la búsqueda preliminar identificó 1.144 artículos potencialmente elegibles, al aplicar los límites de búsqueda y los criterios de selección; quedaron 36 estudios En los cuales eran 21 ECAs y 15 ECC seleccionados (7-9,11-23,25,27,29,31-34,37-40,43-45,61,73$75,82-84)(\mathrm{n}=2.318$ pacientes $)$. Ocho artículos fueron

TABLA II. ESCALA CUALITATIVA DE VAN TULDER

\begin{tabular}{cl}
\hline $\begin{array}{c}\text { Evidencia } \\
\text { sólida }\end{array}$ & $\begin{array}{l}\text { Proporcionada por hallazgos } \\
\text { generalmente consistentes en múltiples } \\
\text { ECAs (3 o más) con calificación de } \\
\text { bajo sesgo }\end{array}$ \\
\hline & $\begin{array}{l}\text { Proporcionado por resultados } \\
\text { generalmente consistentes en 1 }\end{array}$ \\
$\begin{array}{c}\text { Evidencia } \\
\text { moderada }\end{array}$ & $\begin{array}{l}\text { puede interpretar como hallazgos } \\
\text { generalmente consistentes en múltiples }\end{array}$ \\
& $\begin{array}{l}\text { ECAs con calificaciones de sesgo } \\
\text { moderado }\end{array}$ \\
\hline $\begin{array}{c}\text { Evidencia } \\
\text { limitada }\end{array}$ & $\begin{array}{l}\text { Proporcionado por hallazgos } \\
\text { consistentes en 1 o más ECAs con alto } \\
\text { riesgo de sesgo }\end{array}$ \\
\hline $\begin{array}{c}\text { Evidencia } \\
\text { contradictoria }\end{array}$ & $\begin{array}{l}\text { Hallazgos no coherentes o } \\
\text { inconsistentes en múltiples ECAs }\end{array}$ \\
\hline Ninguna & Ningún ECA encontrado \\
evidencia &
\end{tabular}


excluidos por los motivos previamente descritos (Tabla III). Todos se realizaron en pacientes con epicondilalgia lateral. Se agruparon en ejercicio terapéutico sólo versus cualquier otra intervención sola $(n=206)$, ejercicio terapéutico dentro de un programa de tratamiento versus cualquier otra intervención sola ( $\mathrm{n}=1.417$ pacientes) y ejercicio terapéutico dentro de un programa de tratamiento comparado con otro programa de tratamiento $(n=695)$, los tamaños de la muestra variaron entre 5 y 198 pacientes con un promedio de 72 pacientes por estudio, el rango de edad de los pacientes estaba entre $18 \mathrm{y}$ 74 años con un promedio de 46 años.

\section{Intervenciones y comparaciones}

Las intervenciones terapéuticas estudiadas fueron: el ejercicio terapéutico sólo y el ejercicio terapéutico junto a otras intervenciones. En base a los resultados de nuestra búsqueda agrupamos los artículos seleccionados en las siguientes comparaciones: ejercicio terapéutico sólo versus otra intervención sola $(15,18,33,61)$, ejercicio terapéutico sólo versus un programa de tratamiento $(7,9,12,17,19,20,29,34,37,39,43,45,73-75)$ y programa de tratamiento que incluya ejercicio terapéutico versus otro programa de tratamiento $(8,11,14,16,21$ 23,25,27,31,38,40,44,82-84).

\section{Mediciones de resultados}

Las medidas de resultado más comúnmente utilizadas en los artículos seleccionados fueron: Dolor mediante la escala de EVA, funcionalidad medida con la escala DASH y la fuerza de agarre medida con un dinamómetro. Todos los artículos seleccionados por nuestra búsqueda $(7-9,11,12,14-$
23,25,27,29,31-34,37-40,43-45,61,73-75,82-84) estudiaron los efectos con un seguimiento máximo de 3 años.

\section{Síntesis y agrupación de artículos incluidos}

El resumen de todos los aspectos metodológicos de los artículos seleccionados se detalla en la tabla IV, a continuación se presentarán los resultados obtenidos.

\section{Ejercicio terapéutico solo versus cualquier otra intervención sola}

Los 4 estudios $n=206$ pacientes $(18,15,33,61)$ investigaron la efectividad del ejercicio terapéutico sólo comparado con una intervención sola en pacientes con EL.

\section{Ejercicio terapéutico dentro de un programa de tratamiento versus cualquier otra intervención sola}

Los 17 estudios $n=1.417$ pacientes $(7,9,12,17,19,20,23$, $26,29,32,34,37,43,45,73-75)$ investigaron la efectividad del ejercicio terapéutico dentro de un programa de tratamiento comparado con una intervención sola en pacientes con EL.

Ejercicio terapéutico dentro de un programa de
tratamiento versus otro programa de tratamiento

Los 15 estudios $n=695$ pacientes $(8,11,14,16,21,22,25$, 27,31,38,40,44,82-84) investigaron la efectividad del ejercicio terapéutico dentro de un programa de tratamiento comparado con una intervención sola en pacientes con EL.

TABLA III. CARACTERÍSTICAS DE ARTÍCULOS EXCLUIDOS

\begin{tabular}{lll}
\hline \multicolumn{1}{c}{ Autor } & \multicolumn{1}{c}{ Publicación } & \multicolumn{1}{c}{ Motivo de exclusión } \\
\hline $\begin{array}{l}\text { Slater et al. } \\
\text { Chesterton et al. }\end{array}$ & $\begin{array}{l}\text { Man Ther 2010, feb 15(1):66-73 } \\
\text { BMC Musculoskelet Disord. 2009;10:156 }\end{array}$ & $\begin{array}{l}\text { Epicondilalgia experimental } \\
\text { Artículo en fase preliminar sin }\end{array}$ \\
$\begin{array}{l}\text { Slater et al. } \\
\text { Olaussen et al. }\end{array}$ & Manual Therapy, 2006 May;11(2):107-17 & Epicondilalgia lateral experimental \\
& BMC Musculoskeletal Disorders 2009, 10:152 & Artículo en fase preliminar sin \\
Coombes et al. & BMC Musculoskelet Disord 2009 Jun 24;10:76 & Artículo en fase preliminar sin \\
& & interpretación de resultados \\
Strujis et al. & Br J Sports Med 2006 July; 40(7): 637-643 & Estudio de costo-efectividad \\
Korthals-de Bos et al. & Pharmacoeconomics 2004;22(3):185-95 & Estudio de costo-efectividad \\
Slater et al. & Pain 2005 Mar; 114(1-2):118-30 & Epicondilalgia lateral experimental
\end{tabular}


TABLA IV. CARACTERÍSTICAS DE ARTÍCULOS INCLUIDOS

\begin{tabular}{|c|c|c|c|c|}
\hline Autor/Año & Condición/Método & Características pacientes & Intervención & Seguimiento/resultados \\
\hline $\begin{array}{l}\text { Stasinopoulos et al. } \\
(2006) \\
(34)\end{array}$ & $\begin{array}{l}\text { MDA: Se crearon por orden } \\
\text { de llegada de los sujetos. } \\
\text { EL con diagnóstico clínico. } \\
\text { Grupo A: Fisioterapia Cyriax. } \\
\text { Grupo B: Programa de } \\
\text { ejercicios. } \\
\text { Grupo C: Terapia de láser }\end{array}$ & $\begin{array}{l}\mathrm{N}=75 \text { pacientes } \\
\text { Grupo } \mathrm{A}: \mathrm{N}=25 \text { pacientes } \\
\text { Grupo } \mathrm{B}: \mathrm{N}=25 \text { pacientes } \\
\text { Grupo } \mathrm{C}: \mathrm{N}=25 \text { pacientes }\end{array}$ & $\begin{array}{l}\text { Intervención; 4semanas. } \\
\text { Grupo A: Fisioterapia de Cyriax (masaje fricción } \\
\text { transverso profundo) por } 10 \text { min seguido por una } \\
\text { manipulación de Mills. } \\
\text { Grupo B: Programa de ejercicios excéntricos, } 3 \text { series } \\
\text { de } 10 \text { repeticiones y elongaciones de } 6 \text { repeticiones } \\
\text { durante } 30 \text { segundos, } 3 \text { veces antes de los ejercicios y } \\
3 \text { veces después. }\end{array}$ & $\begin{array}{l}\text { Seguimiento: } 6 \text { meses } \\
\text { - Dolor (EVA). } \\
\text { - Función (EVA) } 0 \mathrm{~cm} \text { no } \\
\text { tiene función y } 10 \mathrm{~cm} \text { toda la } \\
\text { función. } \\
\text { - Grip libre de dolor } \\
\text { (Dinamómetro de mano } \\
\text { ajustable Jamar) }\end{array}$ \\
\hline
\end{tabular}

\begin{tabular}{ll}
\hline Dreschler et al. & MDA; No se reporta como se \\
$(1997)$ & realizó la aleatorización. \\
EL con diagnóstico clínico, & comparando 2 tratamientos \\
& excéntricos. \\
& Grupo A: Movilización neural \\
& Grupo B: Tratamiento \\
& estándar \\
\hline Blanchette et al. & MDA: se realizó por un \\
$(2011)$ & método de números en una \\
$(17)$ & urna. \\
& EL con diagnóstico clínico. \\
& Grupo A: Movilización \\
& aumentada de tejido blando. \\
& (MATB) \\
& Grupo B: Consejos \\
& ergonómicos, elongaciones y \\
& en primera etapa AINES \\
& MDA: Se aleatorizó \\
& por bloques y sistema \\
& computarizado. \\
& EL con diagnóstico clínico. \\
& Grupo A: consejos para \\
& realizar ejercicios en casa. \\
& Grupo B: consejos para el \\
& paciente (esperar y ver) \\
&
\end{tabular}

\section{$\mathrm{N}=18$ pacientes} Grupo A: $\mathrm{N}=8$ pacientes $\mathrm{Edad}=30-57$ años, Promedio 46.4 años. Grupo B: $N=10$ pacientes

Edad= $34-53$ años. Promedio 45.5 años

$\mathrm{N}=30$ pacientes Grupo A: $N=15$ pacientes Edad: Promedio 47 años SD: 10 años Grupo B: $N=15$ pacientes Edad: promedio 46 años SD: 10 años

Grupo C: Láser policromático no coherente de 480 $3400 \mathrm{~nm}$, densidad de $2,4 \mathrm{~J} / \mathrm{cm}^{2}$ durante 6 minutos en la zona del epicóndilo

\section{Intervención: 8 semanas, 2 veces al día.}

Grupo A:Movilización del nervio radial

Grupo B: US 1.0 a $1.5 \mathrm{w} / \mathrm{cm}^{2}$ por $5 \mathrm{~min}$. A $3 \mathrm{MHz}$,

seguido de masaje fricción transversal profundo 3

veces por minuto, elongaciones 5 a 10 repeticiones

por 30 segundos y ejercicios de fuerza de los

extensores 3 series de 15 repeticiones ( fue incluido para los ejercicios en casa)

\section{Intervención: 5 semanas}

Grupo A: MATB dos veces por semana, con un instrumento capacitado para mover a lo largo de la estructura músculo tendinosa.

Grupo B: Consejos ergonómicos para el computador, elongaciones de flexores y extensores de muñeca por 30 segundos, 6 veces al día, AINES y hielo

Seguimiento: 3 meses
- Estatus ocupacional
- Estatus recreacional
- Test de tensión neural
- Fuerza del grip (dinamómetro
de mano)

Seguimiento: 3 meses
- Dolor (EVA)
- Grip libre de dolor
(Dinamómetro de mano
JAMAR)
- Evolución de los pacientes
evaluados (PRTEE y EVA)

\begin{tabular}{|c|c|}
\hline $\mathrm{N}=81$ pacientes & Intervención: 3 meses \\
\hline Grupo A: $N=40$ pacientes & Grupo A: consejos de actividad, ejercicios de \\
\hline promedio: 40 años SD 8.1 & extensores en casa con carga progresiva (envase \\
\hline Grupo $B: N=41$ pacientes & de plástico con agua) $1 \mathrm{~kg}$ para mujeres $2 \mathrm{~kg}$ para \\
\hline promedio:47.4 años SD:8.6 & hombres, se incrementaba por semana realizándolo en \\
\hline & $\begin{array}{l}3 \text { series de } 15 \text { repeticiones por dia } \\
\text { Grupo B: se aconsejo de la condición del paciente, el }\end{array}$ \\
\hline & uso de su extremidad y esperar y ver \\
\hline
\end{tabular}

Seguimiento: Posterior a los 3

meses de tratamiento

- Dolor con MVC (EVA)

- Dolor con EMM (EVA)

- Fuerza muscular (dinamómetro chatillón MSE100)

- Función (DASH)

- Calidad de vida (GQL)

- Bienestar (Likert)

Newcomer et al. (2001)

(40)

\section{MDA: No describe el método $\quad \mathrm{N}=39$ pacientes} de aleatorización. EL con diagnóstico clínico. Grupo A: inyección de corticoides (placebo) + ejercicios de criomasaje + elongaciones + ejercicios de

fortalecimiento

Grupo B: Inyección de corticoides (hydrocloridrato de bubivacaína y betametasona)+ ejercicios de criomasaje + elongaciones + ejercicios de fortalecimiento

Kochar et al. MDA: No menciona el método de aleatorización.

(2002) EL con diagnóstico clínico Grupo A: movilización de Mulligan + elongaciones + ejercicios

Grupo B: US

Grupo C: evolución de la condición sin intervenciones Grupo A: N= 19 pacientes Promedio edad: 44.6 años SD:7.6 Grupo B: $\mathrm{N}=20$ pacientes Promedio edad: 46.0 años SD: 7.0

\section{Intervención: 6 semanas}

Grupo A: Inyección corticoides (placebo) $5 \mathrm{ml} \mathrm{de}$ $0.25 \%$ hidrocloridrato de bubivacaína, ejercicios de criomasaje 3 veces por día 5 a 7 minutos, ejercicios de elongación de los músculos extensores y flexores 30 segundos, ejercicios de fortalecimientos 3 series de 15 repeticiones con bandas elásticas.

Grupo B: Inyección corticoides $5 \mathrm{ml}$ de $0.25 \%$ de hydrocloridrato de bubivacaína y betametasona $6 \mathrm{mg} /$ $\mathrm{ml}$, ejercicios de criomasaje 3 veces por día 5 a 7 minutos, ejercicios de elongación de los músculos extensores y flexores 30 segundos, ejercicios de fortalecimientos 3 series de 15 repeticiones con bandas elásticas

\section{$\mathrm{N}=66$ pacientes $\quad$ Intervención: 12 semanas}

Grupo $\mathrm{A}: \mathrm{N}=23$ pacientes promedio edad: 40 años SD: 9

Grupo B: $N=23$ pacientes promedio edad: 45 años SD: 12

Grupo C: $N=20$ pacientes Promedio edad: 38 años SD: 9
$1.5 \mathrm{w} / \mathrm{cm}^{2}$ con pulso de 1 a $5 \mathrm{~ms}$, ejercicio de elongaciones de los músculos extensores de muñeca, ejercicios progresivos de resistencia isométrica 5 a 10 segundos, ejercicios concéntrico y excéntricos 10 repeticiones 3 veces al día.

Grupo B: US a $3 \mathrm{MHz}, 1.5 \mathrm{w} / \mathrm{cm}^{2}$ con pulso de 1 a $5 \mathrm{~ms}$.

Grupo C: evolución de la condición sin intervenciones
Seguimiento: 6 meses

- Dolor (EVA)

- Función (cuestionario funcional de dolor)

- Fuerza del grip (dinamómetro NK biotechnical)
Seguimiento: Posterior a 12

semanas.

- Dolor (EVA)

- Prueba del peso

- Fuerza del grip (dinamómetro)

- Evaluación del estatus del paciente (encuesta simple) 
TABLA IV. CARACTERÍSTICAS DE ARTÍCULOS INCLUIDOS (CONT.)

\begin{tabular}{|c|c|c|c|c|}
\hline Autor/Año & Condición/Método & Características pacientes & Intervención & Seguimiento/resultados \\
\hline $\begin{array}{l}\text { Nilsson et al. } \\
\text { (2007) } \\
\text { (25) }\end{array}$ & $\begin{array}{l}\text { MDA: No se menciona como } \\
\text { se aleatorizó. } \\
\text { EL con diagnóstico clínico. } \\
\text { Grupo A: programa de } \\
\text { ejercicios en casa } \\
\text { Grupo B: inyecciones de } \\
\text { corticoides }\end{array}$ & $\begin{array}{l}\mathrm{N}=78 \text { pacientes } \\
\text { Grupo A: } \mathrm{N}=51 \text { pacientes } \\
\text { Rango de edad: } 32-74 \text { años } \\
\mathrm{X}: 47.9 \\
\text { Grupo B: } \mathrm{N}=27 \text { pacientes } \\
\text { Rango de edad: } 36-67 \text { años } \\
X: 48.2\end{array}$ & $\begin{array}{l}\text { Intervención: } 16 \text { semanas } \\
\text { Grupo A: Programa de ejercicios en casa con bandas } \\
\text { elásticas, ejercicio excéntrico y elongaciones } 15 \\
\text { minutos } 3 \text { veces al día y educación ergonómica. } \\
\text { Grupo B: inyección de corticoides, AINES, gel }\end{array}$ & $\begin{array}{l}\text { Seguimiento: Posterior a las } 16 \\
\text { semanas } \\
\text { - Dolor (EVA) } \\
\text { - Función (PRFEQ) } \\
\text { - Fuerza grip (GRIPPIT) }\end{array}$ \\
\hline $\begin{array}{l}\text { Coff et al. } \\
\text { (2009) } \\
(9)\end{array}$ & $\begin{array}{l}\text { MDA: No menciona como se } \\
\text { aleatorizó } \\
\text { EL con diagnóstico clínico. } \\
\text { Grupo A: fisioterapia inter X } \\
\text { + masaje friccional profundo } \\
\text { + US + elongaciones y } \\
\text { fortalecimiento } \\
\text { Grupo B: masaje } \\
\text { friccional profundo + } \\
\text { US + elongaciones y } \\
\text { fortalecimiento }\end{array}$ & $\begin{array}{l}\mathrm{N}=26 \text { pacientes } \\
\text { Grupo } A: N=13 \text { pacientes } \\
\text { Grupo } B: N=13 \text { pacientes }\end{array}$ & $\begin{array}{l}\text { Intervención: } 3 \text { semanas } \\
\text { Grupo A: modalidad de frecuencia eléctrica inter X } \\
\text { por } 15 \text { minutos, masaje fricción transversal profundo, } \\
\text { US por } 5 \text { minutos, elongación de músculos extensores } \\
\text { de muñeca y ejercicios de fortalecimiento. } \\
\text { Grupo B: masaje fricción transversal profundo, US } \\
\text { por } 5 \text { minutos, elongación de músculos extensores de } \\
\text { muñeca y ejercicios de fortalecimiento }\end{array}$ & $\begin{array}{l}\text { Seguimiento: } 9 \text { meses } \\
\text { - Dolor (EVA) } \\
\text { - Funcionalidad (PRTEEQ) } \\
\text { - Fuerza del grip (Dinamómetro) }\end{array}$ \\
\hline $\begin{array}{l}\text { Bisset et al. } \\
\text { (2006) } \\
(23)\end{array}$ & $\begin{array}{l}\text { MDA: Se aleatorizó a través } \\
\text { de vía telefónica } \\
\text { Dg clínico de codo tenista de } \\
\text { un mínimo de } 6 \text { semanas. } \\
\text { Grupo A: FST + } \\
\text { manipulación de codo + } \\
\text { ejercicio terapéutico. } \\
\text { Grupo B: inyecciones de } \\
\text { corticoides. } \\
\text { Grupo C: ver y esperar } \\
\text { (control) }\end{array}$ & $\begin{array}{l}\mathrm{N}=198 \text { pacientes } \\
\mathrm{DS}=18 \text { a } 65 \text { años } \\
\text { Grupo } \mathrm{A}: \mathrm{N}=67 \text { pacientes } \\
\text { Grupo } \mathrm{B}: \mathrm{N}=65 \text { pacientes } \\
\text { Grupo } \mathrm{C}: \mathrm{N}=66 \text { pacientes }\end{array}$ & $\begin{array}{l}\text { Intervención; } 6 \text { semanas } \\
\text { Grupo A: Sesiones de fisioterapia de } 30 \mathrm{~min} \text { durante } \\
6 \text { semanas previamente manipulación de codo y } \\
\text { ejercicio terapéutico. } \\
\text { Grupo B: Inyecciones de corticoides local } 1 \text { cantidad } \\
\text { de } 1 \mathrm{ml} \text { de lidocaína al } 1 \% \text { con } 10 \mathrm{mg} \text { de acetato de } \\
\text { triamcinolona en } 1 \mathrm{ml} . \\
\text { Grupo C: ver y esperar }\end{array}$ & $\begin{array}{l}\text { Seguimiento: } 52 \text { semanas } \\
\text { - Mejora global sin dolor (escala } \\
\text { tipo likert) } \\
\text { - Fuerza de agarre } \\
\quad \text { (dinamómetro) } \\
\text { - Dolor (EVA) } \\
\text { - Funcionalidad (cuestionario } \\
\text { libre de dolor) }\end{array}$ \\
\hline $\begin{array}{l}\text { Stergioulas et al. } \\
\text { (2007) } \\
(14)\end{array}$ & $\begin{array}{l}\text { MDA: no se describe como se } \\
\text { aleatorizó. } \\
\text { Dg. EL de menos de } 5 \\
\text { semanas. } \\
\text { Grupo A: láser + ejercicios } \\
\text { pliométricos. } \\
\text { Grupo B: laser apagado + } \\
\text { ejercicios pliométricos }\end{array}$ & $\begin{array}{l}\mathrm{N}: 50 \text { pacientes } \\
\text { Grupo A: N: } 25 \text { pacientes. } \\
\text { (16 hombres y } 9 \text { mujeres ) } \\
\text { Grupo B: N: } 25 \text { pacientes. } \\
\text { (15 hombres y } 10 \text { mujeres) }\end{array}$ & $\begin{array}{l}\text { Intervención: } 8 \text { semanas } \\
\text { Grupo A: Láser Ga- As, de } 904 \mathrm{~nm} \text {, continuo, } 50 \\
\mathrm{~Hz}, 40 \mathrm{~mW} \text {, área } 0.5 \mathrm{~cm}^{2} \text { al } 50 \% \text { del ciclo, } 2.4 \mathrm{~J} / \mathrm{cm} \\
2 \text { por } 30 \text { seg. } 5 \text { series de } 8 \text { repeticiones de ejercicios } \\
\text { polimétricos lentos y progresivos de los músculos } \\
\text { extensores de muñeca en cada sesión con un intervalo } \\
\text { de un minuto de descanso. } \\
\text { Grupo B: láser apagado } \\
5 \text { series de } 8 \text { repeticiones de ejercicios polimétricos } \\
\text { lentos y progresivos de los extensores de muñeca en } \\
\text { cada sesión con un intervalo de un min de descanso }\end{array}$ & $\begin{array}{l}\text { Seguimiento: } 8 \text { semanas } \\
\text { - Dolor en reposo y a la } \\
\text { palpación del epicóndilo (EVA) } \\
\text { - Fuerza de Agarre } \\
\text { (dinamómetro) } \\
\text { - ROM } \\
\text { - Prueba del peso (apoyada en } \\
\text { una mesa con el hombro en } \\
60^{\circ} \text { flexión, codo extendido } \\
\text { por completo y pronación } \\
\text { de antebrazo, el pcte levanta } \\
\text { la mano en la extensión de } \\
\text { muñeca sin dolor con un peso } \\
\text { de } 1,2 \text { y } 3 \text { kilos) }\end{array}$ \\
\hline $\begin{array}{l}\text { Struijs et al. } \\
\text { (2003) } \\
(37)\end{array}$ & $\begin{array}{l}\text { MDA: Por datos } \\
\text { demográficos. } \\
\text { Dg EL con quejas de menos } \\
\text { de } 6 \text { semanas y no más de } 6 \\
\text { meses. } \\
\text { Grupo A: manipulación de } \\
\text { muñeca. } \\
\text { Grupo B: US + masaje de } \\
\text { fricción + elongación + } \\
\text { fortalecimiento muscular }\end{array}$ & $\begin{array}{l}\mathrm{N}: 31 \text { pacientes. } \\
\text { Grupo A: } \mathrm{N}: 15 \text { pacientes } \\
\text { Grupo B: } \mathrm{N}: 16 \text { pacientes }\end{array}$ & $\begin{array}{l}\text { Intervención: } 6 \text { semanas } \\
\text { Grupo A: } 9 \text { sesiones de } 2 \text { veces por semana de } \\
\text { maniobras de manipulación } \\
\text { Grupo B: } 9 \text { sesiones ( } 3 \text { sesiones la primera semana, } \\
2 \text { sesiones durante la segunda semana y una sesión } \\
\text { por semana durante las } 4 \text { semanas restantes) US } \\
\text { (Sonopuls 590) pulsado } 7^{1 / 2} \text { por minuto. (20\% ciclo de } \\
\text { trabajo), } 2 \mathrm{~W} / \mathrm{cm}^{2} \text {, masaje de fricción por } 10 \text { minutos, } \\
\text { fortalecimiento muscular y ejercicio de elongación } \\
\text { + ejercicios en casa dos veces por día (movimiento } \\
\text { contra resistencia, ejercicios de rotación) }\end{array}$ & $\begin{array}{l}\text { Seguimiento: } 6 \text { semanas } \\
\text { - Dolor (EVA) } \\
\text { - Fuerza de Agarre } \\
\text { (dinamómetro) } \\
\text { - Dolor a la palpación (presión } \\
\text { por kg / } \mathrm{cm}^{2} \text { Threshold meter) } \\
\text { - Flexo-extensión (goniómetro) }\end{array}$ \\
\hline $\begin{array}{l}\text { Pienimaki et al. } \\
\text { (1996) } \\
\text { (7) }\end{array}$ & $\begin{array}{l}\text { MDA: No describe como se } \\
\text { realizó la aleatorización. } \\
\text { Dg clínico EL } \\
\text { Grupo A: Elongación } \\
\text { + ejercicios de } \\
\text { acondicionamiento muscular } \\
\text { con } 4 \text { intensidades. } \\
\text { Grupo B: US pulsado }\end{array}$ & $\begin{array}{l}\mathrm{N}: 39 \text { pacientes } \\
\text { (14 hombres, } 25 \text { mujeres) } \\
\mathrm{X}: 42,3 \text { años } \\
\text { SD: } 5,4 \text { años } \\
\text { Rango: } 31-53 \text { años } \\
\text { Grupo A: } \mathrm{N}: 20 \text { pacientes } \\
\text { Grupo B: } \mathrm{N}: 19 \text { pacientes }\end{array}$ & $\begin{array}{l}\text { Intervención: } 8 \text { semanas } \\
\text { Grupo A: ejercicios de elongación progresivos, } \\
\text { repetitivos lentos de muñeca con brazo extendido } \\
\text { por } 30 \text { segundos, acondicionamiento muscular una } \\
\text { vez por semana con banda elástica y un bastón. El } \\
\text { programa ejercicios consiste de } 4 \text { a } 6 \text { veces diarias } \\
\text { en el hogar cada uno de los programas incluía } 10 \\
\text { repeticiones en } 2 \text { a } 3 \text { series para cada ejercicio. } \\
\text { Grupo B: US pulsado } 0.3 \text { a } 0.7 \mathrm{w} / \mathrm{cm}^{2}, 2 \text { a } 3 \text { veces a } \\
\text { la semana, relación de pulso } 1: 5 \text {, duración de pulso } \\
\text { de } 2 \mathrm{~ms}, 1 \mathrm{MHz} \text { de Frecuencia, área } 5 \mathrm{~cm}^{2} 10 \text { a } 15 \\
\text { minutos }\end{array}$ & $\begin{array}{l}\text { Seguimiento } 8 \text { semanas } \\
\text { - Dolor (EVA) } \\
\text { - Fuerza de agarre } \\
\quad \text { (dinamómetro) }\end{array}$ \\
\hline
\end{tabular}


TABLA IV. CARACTERÍSTICAS DE ARTÍCULOS INCLUIDOS (CONT.)

\begin{tabular}{|c|c|c|c|c|}
\hline Autor/Año & Condición/Método & Características pacientes & Intervención & Seguimiento/resultados \\
\hline $\begin{array}{l}\text { Nagrale et al. } \\
(2009) \\
(31)\end{array}$ & $\begin{array}{l}\text { MDA: no describe como. } \\
\text { Dg clínico de EL con } \\
\text { síntomas de más de un mes. } \\
\text { Grupo A: masaje friccional } \\
\text { transverso profundo con } \\
\text { manipulación de Mills. }\end{array}$ & $\begin{array}{l}\text { N: } 60 \text { pacientes. } \\
\text { Rango: } 30 \text { a } 60 \text { años } \\
\text { Grupo A: N: } 30 \text { pacientes. } \\
\text { Grupo B: N: } 30 \text { pacientes. }\end{array}$ & $\begin{array}{l}\text { Intervención: } 1 \text { mes } \\
\text { Grupo A: } 10 \text { min de masaje transversal profundo } \\
\text { de cyriax, pcte sentado, flexión de } 90^{\circ} \text { codo con } \\
\text { supinación de antebrazo, tratante estabiliza muñeca y } \\
\text { antebrazo mientras con la otra mano le proporciona } \\
\text { el masaje en el epicóndilo lateral + manipulación de }\end{array}$ & $\begin{array}{l}\text { Seguimiento: } 8 \text { mes } \\
\text { - Dolor (EVA) } \\
\text { - Fuerza de agarre } \\
\quad \text { (dinamómetro) } \\
\text { - Estado Funcional (escala para } \\
\quad \text { codo de tenista TEFS) }\end{array}$ \\
\hline
\end{tabular}

Grupo B: iontoforesis + $\quad$ Mills con pcte sentado en $90^{\circ}$ de abducción + rotación

ejercicio supervisado interna se estabiliza la flexión de muñeca del paciente

y pronación y la otra mano en el olecranon.

Grupo B: iontoforesis (Fc $1 \mathrm{MHz}, \mathrm{I} 0,8 \mathrm{~W} / \mathrm{cm}^{2}$ )

con gel diclofenaco en el área del epicóndilo lateral por 5 minutos + ejercicio supervisado: elongación estático del músculo extensor radial corto del carpo + fortalecimiento excéntrico de músculos extensores de muñeca+ elongación estático por 30 a 45 seg con 6 repeticiones por $30 \mathrm{seg}$ de descanso en cada serie

\begin{tabular}{ll}
\hline Struijs et al. & MDA: Secuencial por \\
$(2004)$ & computador. \\
& Dg clínico de EL por más de \\
& 6 meses. \\
& Grupo A: US pulsado + \\
& masaje friccional + protocolo \\
& de ejercicios para el hogar. \\
& Grupo B: Brace. \\
& Grupo C: combinación de \\
& ambos
\end{tabular}

$\mathrm{N}: 180$ pacientes. Grupo A: N: 56 pacientes. SD: 43 años Grupo B: N: 68 pacientes. SD: 46 años Grupo C: N: 56 pacientes SD: 47 años ambos

\section{Intervención: 6 semanas}

Grupo A: US pulsado durante 7.5 minutos + masaje friccional de 5 a 10 minutos + elongaciones de extensores de muñeca durante 30 segundos y ejercicios de fortalecimiento contra resistencia de flexores y extensores de muñeca 2 a 3 series de 10 repeticiones, 2 veces al día.

Grupo B: brace de uso continuo

Grupo C: brace de uso continuo+ US pulsado durante

7.5 minutos + masaje friccional de 5 a 10 minutos + elongaciones de extensores de muñeca durante 30 segundos y ejercicios de fortalecimiento contra resistencia de flexores y extensores de muñeca 2 a 3 series de 10 repeticiones, 2 veces al día

\begin{tabular}{ll}
\hline Ho et al. & MDA: aleatorización con \\
$(2007)$ & edad y género \\
$(12)$ & Dg clínica de EL durante 3 \\
& meses. \\
& Grupo A: terapia de \\
& microcorriente + ejercicio. \\
& Grupo B: Ejercicios
\end{tabular}

Chung et al. (2004) (22)

\section{Intervención: 3 semanas}

Grupo A: Terapia de microcorriente de 0.3 a $30 \mathrm{~Hz}$ de frecuencia, $50 \%$ duty cicle + un programa de ejercicios para el hogar, de fortalecimiento isotónico y elongaciones tanto de flexores y extensores de muñeca.

Grupo B: Programa de ejercicios para el hogar, de fortalecimiento isotónico y estiramiento de flexores y extensores de muñeca

N: 60 pacientes
Grupo A: N: 29 pacientes
Grupo B: N: 31 pacientes

Intervención: 3 semanas

Grupo A: US pulsado de 2000 pulsos densidad de energía $0.03-0.17 \mathrm{~mJ} / \mathrm{mm} 23$ sesiones por semana +

MDA: Aleatorización por
numeración par e impar.
Dg clínico de EL
Grupo A: US pulsado + el de
antebrazo elongación.
Grupo B: US pulsado placebo
+ estiramiento de antebrazo

$\mathrm{N}$ : 16 pacientes. (3 hombres y 13 mujeres) $\mathrm{X}: 45,3$ años. SD: 30 a 50 años

Grupo B: N: 31 pacientes protocolo de elongación de antebrazo (extensor radial corto del carpo) consiste en 4 repeticiones durante 20 segundos por 4 veces al día.

Grupo B: US pulsado de 2000 pulsos densidad de energía $0.03-0.17 \mathrm{~mJ} / \mathrm{mm}^{2} 3$ sesiones por semana (placebo) + protocolo de elongación de antebrazo (extensor radial corto del carpo) consiste en 4 repeticiones durante 20 segundos por 4 veces al día

\begin{tabular}{lll}
\hline $\begin{array}{l}\text { Pienimaki et al. } \\
(1998)\end{array}$ & $\begin{array}{l}\text { MDA: aleatorización } \\
\text { mediante muchos dibujos }\end{array}$ & N: 30 pacientes. \\
Grupo A: N: 16 pacientes \\
& $\begin{array}{l}\text { Dg clínico de EL } \\
\text { Grupo A: 4 pasos de }\end{array}$ & \\
& ejercicios progresivos $\mathrm{B}: \mathrm{N}: 14$ pacientes \\
& Grupo B: U pulsado & \\
\hline Hernández et al. & Programa informático. & $\mathrm{N}: 46$ pacientes. \\
$(2006)$ & Dg clínico de EL & X: 44.9 años \\
$(21)$ & Grupo A: Crioterapia & SD: $20-60$ años) \\
& Grupo B: Iontoforesis & Grupo A: N: 7pacientes \\
& Grupo C: Láser & Grupo B: N: 2 pacientes \\
& Grupo D: Ultrasonoterapia & Grupo C: $\mathrm{N}: 14$ pacientes. \\
& Grupo E: US & Grupo D: N: 16 pacientes \\
& & Grupo E: $\mathrm{N}: 7$ pacientes.
\end{tabular}

Grupo A: se realizaba 4 pasos de ejercicios

progresivos de stretching de brazo.

Grupo B: Se aplicó US pulsado en la zona del epicóndilo lateral con un pulso de 1:5, con una dosis de $0,5 \mathrm{~W} / \mathrm{cm}^{2}$ durante 15 sesiones

\section{Intervención: 2 meses}

Grupo A: 10 sesiones de crioterapia, $\mathrm{T}^{\circ} 4^{\circ} \mathrm{x}$ dos minutos

Grupo B: 10 sesiones de iontoforesis con diclofenaco $\left(1 \mathrm{~cm}^{3}\right), 1-2 \mathrm{mAx} 16$ minutos

Grupo C: 10 sesiones de láser con una frecuencia de $700 \mathrm{~Hz}$ x 14 minutos

Grupo D: 10 sesiones de ultrasonoterapia con gel antiinflamatorio ketoprofeno o diclofenaco de $0.6 \mathrm{~W} /$ $\mathrm{cm}^{2}$, Frecuencia de $3.3 \mathrm{MHz}$ x 7.3 minutos.

Grupo E: 10 sesiones de US pulsátil de $0.4 \mathrm{~W} / \mathrm{cm}^{2} \mathrm{x}$
Seguimiento: 1 año

- Intensidad de dolor (escala numérica)

- Severidad de la queja de los pacientes (escala numérica de 11 puntos)

- Cuestionario funcional libre de dolor (PFFQ)

- Fuerza de agarre (dinamómetro)

- Mejoría global (escala de 6 puntos)

Seguimiento: 3 semanas

- Dolor (EVA)

- Umbral de mecánica de dolor (algómetro)

- Fuerza máxima de agarre (dinamómetro)

- Fuerza libre de dolor (dinamómetro)

\section{Seguimiento: 3 meses \\ - Dolor (EVA) \\ - Fuerza de agarre (dinamómetro)}

Seguimiento: 36 meses

- Dolor en reposo (EVA)

- Dolor bajo tensión (EVA) 
TABLA IV. CARACTERÍSTICAS DE ARTÍCULOS INCLUIDOS (CONT.)

\begin{tabular}{|c|c|c|c|c|}
\hline Autor/Año & Condición/Método & Características pacientes & Intervención & Seguimiento/resultados \\
\hline $\begin{array}{l}\text { Martínez- Silvestrini } \\
\text { et al. } \\
\text { (2005) } \\
(74)\end{array}$ & $\begin{array}{l}\text { MDA: aleatorización por } \\
\text { género y trabajo } \\
\text { Dg Clínico de EL } \\
\text { Grupo A: Stretching } \\
\text { Grupo B: stretching + } \\
\text { fortalecimiento concéntrico } \\
\text { Grupo C: stretching + } \\
\text { fortalecimiento excéntrico }\end{array}$ & $\begin{array}{l}\text { N: } 94 \text { pacientes. } \\
\text { ( } 50 \text { hombres y } 49 \text { mujeres) } \\
\text { SD: } 45.5+/-7.7 \text { años } \\
\text { Grupo A: } \\
\text { N: } 33 \text { pacientes } \\
\text { X: } 43.1 \text { años } \\
\text { Grupo B: } \\
\text { N: } 30 \text { pacientes } \\
\text { X: } 47.0 \text { años } \\
\text { Grupo C: } \\
\text { N: } 31 \text { pacientes } \\
\text { X: } 46.6 \text { años }\end{array}$ & $\begin{array}{l}\text { Intervención: } 6 \text { semanas } \\
\text { Grupo A: } 2 \text { veces al día, } 3 \text { repeticiones de } 30 \text { segundos } \\
\text { de estiramiento en extensores de muñeca con } 30 \\
\text { segundos de descanso. } \\
\text { Grupo B: } 2 \text { veces al día, } 3 \text { repeticiones de } 30 \text { segundos } \\
\text { de estiramiento en extensores de muñeca con } 30 \\
\text { segundos de descanso }+3 \text { series de } 10 \text { repeticiones } \\
\text { una vez al día de fortalecimiento concéntrico de } \\
\text { extensores de muñeca con } 2 \text { a } 5 \text { minutos de descanso } \\
\text { entre cada serie } \\
\text { Grupo C: } 2 \text { veces al día, } 3 \text { repeticiones de } 30 \text { segundos } \\
\text { de elongación en extensores de muñeca con } 30 \\
\text { segundos de descanso }+3 \text { series de } 10 \text { repeticiones } \\
\text { una vez al día de fortalecimiento excéntrico de } \\
\text { extensores de muñeca con } 2 \text { a } 5 \text { minutos de descanso } \\
\text { entre cada serie }\end{array}$ & $\begin{array}{l}\text { Seguimiento: } 6 \text { semanas } \\
\text { - Dolor (EVA) } \\
\text { - Funcionalidad de miembro } \\
\text { superior (cuestionario DASH) } \\
\text { - Fuerza de Agarre } \\
\text { (dinamómetro) }\end{array}$ \\
\hline $\begin{array}{l}\text { Radpasand et al. } \\
(2009) \\
(16)\end{array}$ & $\begin{array}{l}\text { MDA: Por tabla de números } \\
\text { aleatorios. } \\
\text { Dg clínico de EL } \\
\text { Grupo A: Manipulación de } \\
\text { alta velocidad y baja amplitud } \\
\text { + corriente galvánica + } \\
\text { Brace+ hielo + ejercicios. } \\
\text { Grupo B: US+ brace + } \\
\text { ejercicios }\end{array}$ & $\begin{array}{l}\text { N: } 5 \text { pacientes ( } 4 \text { hombres } \\
\text { y } 1 \text { mujer) } \\
\text { SD: } 21 \text { a } 65 \text { años } \\
\text { Grupo A: } 3 \text { pacientes } \\
\text { (hombres) } \\
\text { Grupo B: } 2 \text { pacientes (1 } \\
\text { mujer y un hombre) }\end{array}$ & $\begin{array}{l}\text { Intervención: } 12 \text { semanas } \\
\text { Grupo A: Manipulación de alta velocidad y baja } \\
\text { amplitud por } 10 \text { minutos } 3 \text { veces por semana por } \\
4 \text { un mes + corriente galvánica + brace continuo + } \\
\text { hielo local por un máximo de } 10 \text { minutos en el sitio } \\
\text { del dolor por } 3 \text { veces al día + ejercicios isométricos } \\
\text { en flexores, extensores, pronadores y supinadores al } \\
\text { final del rango de movimiento por } 10 \text { segundos, } 10 \\
\text { repeticiones como máximo } 2 \text { veces al día } \\
\text { Grupo B: US pulsado de baja intensidad con una } \\
\text { frecuencia de } 3 \mathrm{MHz}, 1.5 \mathrm{~W} / \mathrm{cm}^{2} \text {, área cabezal } 2 \mathrm{~cm}^{2} \\
\text { x } 8 \text { minutos + brace continuo + ejercicios isométricos } \\
\text { en flexores, extensores, pronadores y supinadores al } \\
\text { final del rango de movimiento por } 10 \text { segundos, } 10 \\
\text { repeticiones como máximo } 2 \text { veces al día }\end{array}$ & $\begin{array}{l}\text { Seguimiento: } 12 \text { semanas } \\
\text { - Dolor (EVA) }\end{array}$ \\
\hline $\begin{array}{l}\text { Verhaar } \\
\text { (1996) } \\
\text { (39) }\end{array}$ & $\begin{array}{l}\text { MDA: Aleatorización a } \\
\text { través de sobres cerrados } \\
\text { enumerados. } \\
\text { Dg clínico de EL. } \\
\text { Grupo A: inyecciones de } \\
\text { corticoides } \\
\text { Grupo B: Masaje Cyriax }\end{array}$ & $\begin{array}{l}\text { N: } 106 \text { pctes ( } 59 \text { hombres } \\
\text { y } 47 \text { mujeres) } \\
\text { X: } 43 \text { años }\end{array}$ & $\begin{array}{l}\text { Intervención: } 6 \text { semanas } \\
\text { Grupo A: } 1 \mathrm{ml} \text { de acetato de triamcinolona, } 1 \% \text { diluida } \\
\text { con } 1 \mathrm{ml} \text { de lidocaína al } 1 \% \text { dentro del tendón del } \\
\text { músculo extensor radial corto del carpo y extensor } \\
\text { de los dedos, } 2 \text { a } 3 \text { inyecciones dependiendo de la } \\
\text { aparición de los síntomas. } \\
\text { Grupo B: un masaje de fricción profunda en } \\
\text { el músculo extensor radial corto del carpo y } \\
\text { manipulación de Mills, } 12 \text { sesiones por } 4 \text { semanas }\end{array}$ & $\begin{array}{l}\text { Seguimiento: } 52 \text { semanas } \\
\text { - Dolor (EVA) } \\
\text { - Fuerza de agarre } \\
\text { (dinamómetro) }\end{array}$ \\
\hline $\begin{array}{l}\text { Manias et al. } \\
\text { (2006) } \\
(44)\end{array}$ & $\begin{array}{l}\text { MDA: aleatorización } \\
\text { secuencial } \\
\text { Dg clínico de EL } \\
\text { Grupo A: programa de } \\
\text { ejercicios + hielo. } \\
\text { Grupo B: programa de } \\
\text { ejercicios }\end{array}$ & $\begin{array}{l}\mathrm{N}: 40 \text { pacientes } \\
\text { Grupo A: } 20 \text { pacientes } \\
\text { Grupo B: } 20 \text { pacientes }\end{array}$ & $\begin{array}{l}\text { Intervención: } 4 \text { semanas } \\
\text { Grupos A: programa de ejercicios excéntricos de } \\
\text { extensores de muñeca } 3 \text { series de } 3 \text { repeticiones y } \\
\text { estiramiento estático de extensor radial corto del } \\
\text { carpo } 6 \text { veces en cada sesión ( } 3 \text { veces antes de los } \\
\text { excéntricos y } 3 \text { veces) después con } 30 \text { segundos de } \\
\text { descanso entre cada una de las repeticiones)+ hielo } \\
\text { por } 10 \text { minutos en el epicóndilo lateral. } \\
\text { Grupos B: Programa de ejercicios excéntricos } \\
\text { de músculos extensores de muñeca ( } 3 \text { series de } \\
3 \text { repeticiones) y elongación estático de músculo } \\
\text { extensor radial corto del carpo } 6 \text { veces en cada sesión } \\
\text { (3 veces antes de los excéntricos y } 3 \text { veces después } \\
\text { con } 30 \text { segundos de descanso entre cada una de las } \\
\text { repeticiones) }\end{array}$ & $\begin{array}{l}\text { Seguimiento: } 16 \text { semanas } \\
\text { - Dolor (EVA) }\end{array}$ \\
\hline $\begin{array}{l}\text { Smidt et al. } \\
\text { (2002) } \\
(43)\end{array}$ & $\begin{array}{l}\text { MDA: Generador de números } \\
\text { aleatorios computarizados. } \\
\text { Dg clínico de EL } \\
\text { Grupo A: Inyección de } \\
\text { corticoides. } \\
\text { Grupo B: FST. } \\
\text { Grupo C: Ver y esperar }\end{array}$ & $\begin{array}{l}\mathrm{N}: 183 \text { pacientes } \\
\text { Grupo A: } \\
\mathrm{N}: 60 \text { pacientes } \\
\text { Grupo B: } \\
\mathrm{N}: 64 \text { pacientes } \\
\text { Grupo C: } \mathrm{N}: 59 \text { pacientes }\end{array}$ & $\begin{array}{l}\text { Intervención: } 6 \text { semanas } \\
\text { Grupo A: Inyección de corticoides de } 1 \mathrm{ml} \text { de acetato } \\
\text { de triamcinolona }(10 \mathrm{mg} / \mathrm{mL}) \text { y } 1 \mathrm{ml} \text { de lidocaína al } \\
2 \% \text {. } \\
\text { Grupo B: } 9 \text { sesiones de US pulsado ( } 20 \% \text { duty cycle, } \\
\mathrm{I} 2 \mathrm{~W} / \mathrm{cm}^{2} \text { por } 7.5 \text { minutos por sesión, masaje cyriax } \\
\text { y programa de ejercicios de elongación de codo y } \\
\text { muñeca con ejercicios ocupacionales por } 6 \text { semanas. } \\
\text { Grupo C: Ver y esperar }\end{array}$ & $\begin{array}{l}\text { Seguimiento: } 52 \text { semanas } \\
\text { - Dolor (EVA) } \\
\text { - Fuerza de agarre } \\
\quad \text { (dinamómetro) }\end{array}$ \\
\hline
\end{tabular}


TABLA IV. CARACTERÍSTICAS DE ARTÍCULOS INCLUIDOS (CONT.)

\begin{tabular}{|c|c|c|c|c|}
\hline Autor/Año & Condición/Método & Características pacientes & Intervención & Seguimiento/resultados \\
\hline $\begin{array}{l}\text { Croisier et al. } \\
(2007) \\
(73)\end{array}$ & $\begin{array}{l}\text { MDA: la aleatorización de } \\
\text { pctes se consideró edad, } \\
\text { género y actividad. } \\
\text { Dg clínico e imagenológico } \\
\text { de EL. } \\
\text { Grupo A: ejercicios } \\
\text { excéntricos. } \\
\text { Grupo B:control }\end{array}$ & $\begin{array}{l}\text { N: } 92 \text { pacientes. } \\
\text { Grupo A: } \\
\text { N: } 46 \text { pacientes. (X: } 40 \\
\text { DS 8) } \\
\text { Grupo B: N: } 46 \text { pacientes } \\
\text { (x: } 38 \text { DS } 8 \text { ) }\end{array}$ & $\begin{array}{l}\text { Intervención: } 9 \text { semanas } \\
\text { Grupo A: Entrenamiento excéntrico isocinético } \\
\text { (extensores de muñeca y supinadores de codo) } 3 \text { veces } \\
\text { a la semana. } \\
\text { Grupo B: hielo, TENS, US, masaje fricción profunda } \\
\text { y stretching }\end{array}$ & $\begin{array}{l}\text { Seguimiento: } 9 \text { semanas } \\
\text { - Dolor (EVA) } \\
\text { - Fuerza de agarre } \\
\quad \text { (dinamómetro) }\end{array}$ \\
\hline $\begin{array}{l}\text { Tyler et al. } \\
(2010) \\
(82)\end{array}$ & $\begin{array}{l}\text { MDA: No describe como se } \\
\text { realizó la aleatorización. } \\
\text { Dg clínico de EL. } \\
\text { Grupo A: Protocolo estándar. } \\
\text { Grupo B: ejercicios. } \\
\text { excéntricos }\end{array}$ & $\begin{array}{l}\text { N: } 21 \text { pctes. } \\
\text { Grupo A: } N: 10 \text { pacientes. } \\
\text { (X: } 51+/-4 \text { años) } \\
\text { Grupo B: } N: 11 \text { pacientes } \\
\text { (X: } 47+/-2 \text { años) }\end{array}$ & $\begin{array}{l}\text { Intervención: } 7 \text { semanas } \\
\text { Grupo A: Calor, elongación de músculos extensores } \\
\text { de muñeca, US, Masaje de fricción, Hielo + } \\
\text { Fortalecimiento isotónico de extensores de muñeca. } \\
\text { Grupo B: Calor, elongaciones de extensores } \\
\text { de muñeca, US, Masaje de fricción Hielo + } \\
\text { fortalecimiento excéntrico de músculos estaba } \\
\text { extensores de muñeca (Usando banda elástica) cada } \\
\text { contracción excéntrica de extensores de muñeca (3 } \\
\text { series de } 15 \text { repeticiones diariamente con } 30 \text { segundos } \\
\text { de descanso) }\end{array}$ & $\begin{array}{l}\text { Seguimiento: } 10 \text { semanas } \\
\text { - Dolor (EVA) } \\
\text { - Fuerza de agarre } \\
\quad \text { (dinamómetro) } \\
\text { - Cuestionario (DASH) }\end{array}$ \\
\hline $\begin{array}{l}\text { Stasinopoulos et al. } \\
2010 \\
(83)\end{array}$ & $\begin{array}{l}\text { MDA: Aleatorizacion por } \\
\text { orden de llegada } \\
\text { Diagnostico clínico de EL. } \\
\text { Grupo A: Programa de } \\
\text { ejercicio en casa } \\
\text { Grupo B: Programa de } \\
\text { ejercicio supervisado }\end{array}$ & $\begin{array}{l}\mathrm{N}=70 \text { pacientes } \\
\text { Grupo A: } \mathrm{N}=35 \text { pacientes. } \\
\text { Edad : } \mathrm{X}=44.38 \text { SD:5.39 } \\
\text { Grupo } \mathrm{B}: \mathrm{N}=35 \text { pacientes } \\
\text { Edad: } 45.72 \text { SD: } 6.21\end{array}$ & $\begin{array}{l}\text { Intervención: } 12 \text { semanas } \\
\text { Grupo A: Ejercicio excéntrico lento y progresivo con } \\
\text { el codo apoyado sobre una superficie en completa } \\
\text { extensión y con pronacion, } 3 \text { series de } 12 \text { repeticiones, } \\
\text { elongación de músculo extensor corto del carpo } \\
\text { realizado } 3 \text { veces antes y después de los ejercicios } \\
\text { excéntricos durante } 30 \text { segundos. } \\
\text { Grupo B: Lo mismo que el anterior pero con } \\
\text { supervisión de un terapeuta }\end{array}$ & $\begin{array}{l}\text { Seguimiento: } 24 \text { semanas } \\
\text { - Dolor (EVA) } \\
\text { - Función (EVA cm) } \\
\text { - Fuerza de agarre } \\
\quad \text { (dinamómetro) }\end{array}$ \\
\hline $\begin{array}{l}\text { Oken } 2008 \text { et al. } \\
\text { (8) }\end{array}$ & $\begin{array}{l}\text { MDA: Aleatorización por } \\
\text { lista numérica. } \\
\text { Diagnóstico clínico de EL. } \\
\text { Grupo A: Grupo de Brace } \\
\text { Grupo B: Grupo de US } \\
\text { Grupo C: Grupo de Láser }\end{array}$ & $\begin{array}{l}\mathrm{N}=59 \text { pacientes } \\
\text { Grupo } \mathrm{A}: \mathrm{N}=20 \text { pacientes. } \\
\text { Edad : } \mathrm{X}=44.5 \mathrm{SD}: 8.9 \\
\text { Grupo } \mathrm{B}: \mathrm{N}=19 \text { pacientes } \\
\text { Edad: } 46.5 \mathrm{SD}: 8.3 \\
\text { Grupo } \mathrm{C}: \mathrm{N}=20 \text { pacientes } \\
\text { Edad: } 45.1 \mathrm{SD}: 8.2\end{array}$ & $\begin{array}{l}\text { Intervención: } 2 \text { semanas } \\
\text { Grupo A: Uso de Brace durante el día por dos } \\
\text { semanas. } \\
\text { Grupo B: Ultrasonido continuo } 1 \mathrm{Mhz}, 1.5 \mathrm{w} / \mathrm{cm}^{2} \text { por } \\
5 \mathrm{~min} \text {. } \\
\text { Grupo C: Láser a baja intensidad, longitud de onda de } \\
632.8 \mathrm{nM} \text { a } 10 \mathrm{mV} \text { por } 10 \text { min. } \\
\text { Los tres grupos recibieron un programa de ejercicios } \\
\text { de elongación y fortalecimiento, extensión y flexión } \\
\text { de muñeca con resistencia, elongación de extensores } \\
\text { de muñeca } 3 \text { series de } 10 \text { repeticiones por } 30 \text { segundos }\end{array}$ & $\begin{array}{l}\text { Seguimiento : } 6 \text { semanas } \\
\text { - Dolor (EVA) } \\
\text { - Fuerza de agarre } \\
\quad \text { (dinamómetro) } \\
\text { - Evaluación de mejora global } \\
\quad \text { (Escala de } 6 \text { puntos) }\end{array}$ \\
\hline $\begin{array}{l}\text { Soderberg et al. } \\
2011 \\
(20)\end{array}$ & $\begin{array}{l}\text { MDA: Aleatorizacion por } \\
\text { bloques. } \\
\text { Dg clínico de EL. } \\
\text { Grupo A: Grupo de ejercicios } \\
\text { Grupo B: Grupo control } \\
\end{array}$ & $\begin{array}{l}\mathrm{N}=42 \text { pacientes } \\
\text { Grupo A: } \mathrm{N}=20 \text { pacientes. } \\
\text { Edad: } \mathrm{X}=48 \mathrm{SD}: 12.4 \\
\text { Grupo } \mathrm{B}: \mathrm{N}=22 \text { pacientes } \\
\text { Edad: } 50 \text { SD: } 10.8\end{array}$ & $\begin{array}{l}\text { Intervención: } 3 \text { semanas } \\
\text { Grupo A: Programa de ejercicios excéntrico en casa, } \\
\text { con el codo apoyado y en flexión de } 70^{\circ}, 2 \text { series de } \\
12 \text { repeticiones. } \\
\text { Grupo B: Programa de ejercicios control }\end{array}$ & $\begin{array}{l}\text { Seguimiento: } 6 \text { semanas } \\
\text { - Dolor (EVA) } \\
\text { - Fuerza de agarre libre de dolor } \\
\quad \text { (dinamómetro) }\end{array}$ \\
\hline $\begin{array}{l}\text { Bisset et al. } \\
(2009) \\
(45)\end{array}$ & $\begin{array}{l}\text { MDA: No se describe el } \\
\text { método de aleatorización. } \\
\text { Dg clínico de EL. } \\
\text { Grupo A: Esperar y ver } \\
\text { Grupo B: Inyección de } \\
\text { corticoides } \\
\text { Grupo C: Movilización y } \\
\text { ejercicios }\end{array}$ & $\begin{array}{l}\text { N=198 pacientes } \\
\text { Grupo EL total: X: } 47.6 \\
\text { Rango: } 28-69 \\
\text { Grupo A: N: } 67 \text { pacientes. } \\
\text { Grupo B: N: } 65 \text { pacientes. } \\
\text { Grupo C: N: } 66 \text { pacientes }\end{array}$ & $\begin{array}{l}\text { Intervención: } 6 \text { semanas } \\
\text { Grupo A:Se realizaron consejos ergonómicos para } \\
\text { modificar actividades que agraven síntomas } \\
\text { Grupo B: Se administro una inyección de } \\
\text { corticoides de } 1 \mathrm{ml} \text { de lidocaína al } 1 \% \text { con } 10 \mathrm{mg} \text { de } \\
\text { triamcinolona en } 1 \mathrm{ml} \text {. } \\
\text { Grupo C: Se realizó movilización con movimiento de } \\
\text { Mulligan y un programa de ejercicio terapéutico }\end{array}$ & $\begin{array}{l}\text { Seguimiento: } 52 \text { semanas } \\
\text { - Movimiento de reacción } \\
\text { extremidad superior (tiempo } \\
\text { de reacción) }\end{array}$ \\
\hline $\begin{array}{l}\text { Stasinopoulos et al. } \\
(2009) \\
(38)\end{array}$ & $\begin{array}{l}\text { MDA: Aleatorización por } \\
\text { orden de llegada. } \\
\text { Dg clínico de EL. } \\
\text { Grupo A: Grupo de ejercicios } \\
\text { más láser de baja frecuencia. } \\
\text { Grupo B: Grupo de ejercicios } \\
\text { más laser policromático no } \\
\text { coherente }\end{array}$ & $\begin{array}{l}\mathrm{N}=50 \text { pacientes } \\
\text { Grupo A: N: } 25 \text { pacientes. } \\
\text { Grupo } \mathrm{B}: \mathrm{N}: 25 \text { pacientes }\end{array}$ & $\begin{array}{l}\text { Intervención: } 4 \text { semanas } \\
\text { Grupo A: Se realizaron programa de ejercicios } \\
\text { excéntrico los extensores de muñeca, } 3 \text { series de } 12 \\
\text { repeticiones, elongaciones } 3 \text { veces antes y después } \\
\text { de la sesión, junto con un láser de Ga-As, longitud } \\
\text { de onda } 904 \mathrm{~nm} \text { a } 50 \mathrm{~Hz} \text { y } 65 \mathrm{~mW} \text { por } 30 \text { seg de } \\
\text { aplicación. } \\
\text { Grupo B: Se realizaron programa de ejercicios } \\
\text { excéntrico de músculos extensores de muñeca, } 3 \\
\text { series de } 12 \text { repeticiones, elongaciones } 3 \text { veces antes } \\
\text { y después de la sesión, junto con láser no coherente } \\
\text { policromático } 480-3400 \mathrm{~nm} \text { a } 40 \mathrm{~mW} / \mathrm{cm}^{2} \text { por } 6 \text { min }\end{array}$ & $\begin{array}{l}\text { Seguimiento: } 16 \text { semanas } \\
\text { - Dolor (EVA) } \\
\text { - Función (Puntuación EVA) } \\
\text { - Fuerza de agarre libre de dolor } \\
\quad \text { (dinamómetro) }\end{array}$ \\
\hline
\end{tabular}


TABLA IV. CARACTERÍSTICAS DE ARTÍCULOS INCLUIDOS (CONT.)

\begin{tabular}{|c|c|c|c|c|}
\hline Autor/Año & Condición/Método & Características pacientes & Intervención & Seguimiento/resultados \\
\hline $\begin{array}{l}\text { Bhardwaj et al. } \\
\text { (2011) } \\
\text { (32) }\end{array}$ & $\begin{array}{l}\text { MDA: Muestreo aleatorio } \\
\text { simple. } \\
\text { Dg clínico de EL. } \\
\text { Grupo A: US+ Movilización } \\
\text { con movimiento. } \\
\text { Grupo B: US+ Terapia de } \\
\text { cyriax } \\
\text { Grupo C: US (grupo control) }\end{array}$ & $\begin{array}{l}\mathrm{N}=60 \text { pacientes } \\
\text { Grupo } \mathrm{A}: \mathrm{N}=20 \text { pacientes. } \\
\text { Grupo } \mathrm{B}: \mathrm{N}=20 \text { pacientes } \\
\text { Grupo } \mathrm{C}: \mathrm{N}=20 \text { pacientes } \\
\text { Rango de edad: } 45-54 \text { años }\end{array}$ & $\begin{array}{l}\text { Intervención: } 4 \text { semanas } \\
\text { Grupo A: Se realizó US por } 10 \text { min. Movilización } \\
\text { con movimiento con el brazo apoyado sobre una } \\
\text { superficie, la técnica era realizada en } 3 \text { series de } 10 \\
\text { repeticiones durante } 6 \text { segundos. } \\
\text { Grupo B: Se realizó US, masaje de fricción transversal } \\
\text { por } 10 \text { min .más manipulación de Mills, } 3 \text { veces por } \\
\text { semana. } \\
\text { Grupo C: Se realizó US pulsado a } 1 \mathrm{Mhz}, 1 \mathrm{w} / \mathrm{cm}^{2} \\
\text { por } 10 \text { min }\end{array}$ & $\begin{array}{l}\text { Seguimiento: } 1 \text { mes } \\
\text { - Dolor (EVA) } \\
\text { - PRFEQ (Funcionalidad) } \\
\text { - Fuerza de agarre } \\
\quad \text { (dinamómetro) }\end{array}$ \\
\hline $\begin{array}{l}\text { Luginbuhl et al. } \\
\text { (2008) } \\
(75)\end{array}$ & $\begin{array}{l}\text { MDA: No se describe el } \\
\text { método de aleatorización. } \\
\text { Dg clínico de EL. } \\
\text { Grupo A: Grupo de banda de } \\
\text { soporte de antebrazo. } \\
\text { Grupo B: Grupo de ejercicios } \\
\text { de fortalecimiento. } \\
\text { Grupo C: Ambos métodos }\end{array}$ & $\begin{array}{l}\mathrm{N}=29 \text { pacientes } \\
\text { Grupo } \mathrm{A}: \mathrm{N}=10 \text { pacientes. } \\
\text { Grupo } \mathrm{B}: \mathrm{N}=9 \text { pacientes } \\
\text { Grupo } \mathrm{C}: \mathrm{N}=10 \text { pacientes } \\
\text { Edad X: } 47 \mathrm{SD} 9 .\end{array}$ & $\begin{array}{l}\text { Intervención: } 6 \text { semanas } \\
\text { Grupo A: Se administró el soporte de banda para } \\
\text { el codo durante el día, cuando se trabaja o realiza } \\
\text { actividades. } \\
\text { Grupo B: Se realizaron programas de ejercicios de } \\
\text { fuerza de agarre con una pelota de tenis } 20 \text { veces } \\
\text { durante } 20 \text { segundos, dos veces al día, ejercicios de } \\
\text { resistencia de extensores de muñeca } 20 \text { segundos, dos } \\
\text { veces al día. } \\
\text { Grupo C: Ambas intervenciones }\end{array}$ & $\begin{array}{l}\text { Seguimiento: } 1 \text { año } \\
\text { - Mejoría (Kirsch y Petrone } \\
\text { score) } \\
\text { - Dolor (Escala de puntuación } \\
\text { en reposo) } \\
\text { - Fuerza de agarre libre de dolo } \\
\quad \text { (dinamómetro) }\end{array}$ \\
\hline $\begin{array}{l}\text { Ajimsha et al. } \\
(2012) \\
(18)\end{array}$ & $\begin{array}{l}\text { MDA: No se describe el } \\
\text { método de Aleatorización. } \\
\text { Dg clínico de EL. } \\
\text { Grupo A: Grupo de liberación } \\
\text { miofascial. } \\
\text { Grupo B: Grupo de control }\end{array}$ & $\begin{array}{l}\mathrm{N}=65 \text { pacientes } \\
\text { Edad X: } 47 \mathrm{SD} 9 . \\
\text { Grupo } \mathrm{A}: \mathrm{N}=10 \text { pacientes. } \\
\text { Grupo } \mathrm{B}: \mathrm{N}=9 \text { pacientes } \\
\text { Grupo } \mathrm{C}: \mathrm{N}=10 \text { pacientes }\end{array}$ & $\begin{array}{l}\text { Intervención: } 4 \text { semanas } \\
\text { Grupo A: se realizó el protocolo de liberación } \\
\text { miofascial en el extensor corto del carpo sobre } \\
\text { el epicóndilo, en el periostio de la ulna y sobre el } \\
\text { radio. Además se posicionó la extremidad sobre una } \\
\text { superficie, con el codo pronado y con una flexión de } \\
15^{\circ} \text { por } 5 \text { minutos por dos repeticiones. } \\
\text { Grupo B: Se realizó terapia de ultrasonido placebo } \\
\text { removiendo el cristal de cuarzo, en el músculo } \\
\text { extensor corto radial del carpo por } 10 \text { minutos }\end{array}$ & $\begin{array}{l}\text { Seguimiento: } 1 \text { año } \\
\text { - PRTEE (funcionalidad y } \\
\text { dolor) }\end{array}$ \\
\hline $\begin{array}{l}\text { Svernlov et al. } \\
\text { (2001) } \\
\text { (33) }\end{array}$ & $\begin{array}{l}\text { MDA: No se describe el } \\
\text { método de aleatorización. } \\
\text { Dg clínico de EL. } \\
\text { Grupo A: Grupo de } \\
\text { Elongación en músculos } \\
\text { extensores del antebrazo. } \\
\text { Grupo B: Grupo de ejercicio } \\
\text { excéntrico de flexores y } \\
\text { extensores del antebrazo }\end{array}$ & $\begin{array}{l}\mathrm{N}=30 \text { pctes. } \\
\text { Grupo } A: N=15 \text { pacientes. } \\
\text { Grupo } B: N=15 \text { pacientes }\end{array}$ & $\begin{array}{l}\text { Intervención: } 12 \text { semanas. } \\
\text { Grupo A: Realizaron estiramientos, el cual consistió } \\
\text { en contracción de los músculos extensores del } \\
\text { antebrazo durante } 10 \text { segundos, relajación durante } \\
2 \text { segundos, estiramiento entre } 15-20 \text { segundos, } 3-5 \\
\text { repeticiones por dos veces al día. } \\
\text { Grupo B: se sometieron a un régimen de ejercicio } \\
\text { excéntrico que consistía en un calentamiento del } \\
\text { antebrazo de músculos extensores y flexores de } \\
\text { muñeca; realizando movimientos sin ningún tipo de } \\
\text { carga durante 2-3 minutos, estiramiento estático 15-30 } \\
\text { segundos por 3-5 veces, ejercicios excéntricos de la } \\
\text { musculatura de antebrazo durante } 10 \text { segundos el cual } \\
\text { se realizaron tres series de } 5 \text { repeticiones con una pesa } \\
\text { y estiramiento estático antes de realizar el ejercicio. } \\
\text { Todos los pacientes se apoyaron con una banda de } \\
\text { soporte de codo }\end{array}$ & $\begin{array}{l}\text { Seguimiento: } 1 \text { año. } \\
\text { - Dolor (EVA) } \\
\text { - Fuerza de prensión } \\
\quad \text { (dinamómetro) }\end{array}$ \\
\hline $\begin{array}{l}\text { Park et al. } \\
(2009) \\
(84)\end{array}$ & $\begin{array}{l}\text { MDA: programa generador } \\
\text { de números aleatorios } \\
\text { Dg clínico de EL. } \\
\text { Grupo A: Grupo de ejercicios } \\
\text { de fortalecimiento isométrico } \\
\text { Grupo B: AINES + ejercicios } \\
\text { de fortalecimiento isométrico }\end{array}$ & $\begin{array}{l}\mathrm{N}=31 \text { pctes } \\
\text { Grupo } \mathrm{A}: \mathrm{N}=16 \text { pacientes. } \\
\text { Grupo } \mathrm{B}: \mathrm{N}=15 \text { pacientes }\end{array}$ & $\begin{array}{l}\text { Intervención: } 4 \text { semanas } \\
\text { Grupo A: se les aplico la terapia inmediatamente en } \\
\text { el momento de la consulta, los cuales se les instruyo } \\
\text { como realizar el ejercicio. } \\
\text { Grupo B: a los que se les administra AINES durante } \\
\text { cuatro semanas y luego realizan el programa de } \\
\text { ejercicios. El programa de ejercicio fue el mismo } \\
\text { para ambos grupos y consistió en fortalecimiento } \\
\text { isométrico, el programa consistía en } 4 \text { series de } \\
50 \text { repeticiones, a cada paciente se le entrego un } \\
\text { calendario para llevar un registro de los días que } \\
\text { realizaba los ejercicios }\end{array}$ & $\begin{array}{l}\text { Seguimiento: } 1 \text { año } \\
\text { - Dolor (EVA) } \\
\text { - MEPS (Funcionalidad) }\end{array}$ \\
\hline
\end{tabular}


TABLA IV. CARACTERÍSTICAS DE ARTÍCULOS INCLUIDOS (CONT.)

\begin{tabular}{|c|c|c|c|c|}
\hline Autor/Año & Condición/Método & Características pacientes & Intervención & Seguimiento/resultados \\
\hline $\begin{array}{l}\text { Viswas et al. } \\
\text { (2012) } \\
\text { (11) }\end{array}$ & $\begin{array}{l}\text { MDA: No se describe el } \\
\text { método de Aleatorización. } \\
\text { Dg clínico de EL. } \\
\text { Grupo A: Grupo de } \\
\text { elongación + fortalecimiento } \\
\text { excéntrico. } \\
\text { Grupo B: Masaje de fricción } \\
\text { profunda + Manipulación } \\
\text { de Mills }\end{array}$ & $\begin{array}{l}\mathrm{N}=20 \text { pacientes } \\
\text { Grupo } A: N=10 \text { pacientes. } \\
\text { Grupo } B: N=10 \text { pacientes }\end{array}$ & $\begin{array}{l}\text { Intervención: } 4 \text { semanas } \\
\text { Grupo A: Consistía en estiramiento estático que se } \\
\text { realiza en sedente con extensión de codo, el antebrazo } \\
\text { en pronación y flexión de la muñeca con desviación } \\
\text { cubital. Respecto a la fuerza de estiramiento se } \\
\text { realizó a tolerancia del paciente. Esta posición de } \\
\text { estiramiento se llevó a cabo durante } 30-45 \text { segundos } \\
\text { y se realizó } 3 \text { veces antes y } 3 \text { veces después de los } \\
\text { ejercicios excéntricos con un total de } 6 \text { repeticiones. } \\
\text { Hubo un intervalo de descanso de } 30 \text { segundos entre } \\
\text { cada elongación. Los ejercicios de fortalecimiento } \\
\text { excéntrico se realizaron en sedente, codo en extensión, } \\
\text { el antebrazo en pronación y la máxima extensión } \\
\text { de muñeca desde esta posición el paciente baja } \\
\text { lentamente la muñeca hacia flexión, con la mano } \\
\text { contralateral se ayuda para volver a la extensión } \\
\text { máxima. Para que el ejercicio fuera bien tolerado } \\
\text { se incrementó la utilización de pesas realizando } 10 \\
\text { repeticiones máximas, tres series de diez repeticiones } \\
\text { durante cada entrenamiento con un intervalo de } \\
\text { descanso de } 1 \text { minuto entre cada serie. Los pacientes } \\
\text { recibieron manual de educación respecto a la } \\
\text { ergonomía y la técnica de modificación de la actividad } \\
\text { para evitar la agravación de los síntomas. } \\
\text { Grupo B: Se le aplicó fisioterapia Cyriax, el cual } \\
\text { consistía en } 10 \text { minutos de masaje de fricción } \\
\text { transversal profunda seguido inmediatamente por } \\
\text { una sola aplicación de la manipulación de Mills. La } \\
\text { fricción transversal profunda de codo se realiza con el } \\
\text { paciente cómodamente en supinación completa y en } \\
90^{\circ} \text { de flexión. Luego de palpar la zona antero lateral } \\
\text { del epicóndilo lateral del húmero, se aplica fricción } \\
\text { transversa profunda con el lado de la punta del pulgar. } \\
\text { La presión se aplica en una dirección posterior a la } \\
\text { unión osteotendinea durante } 10 \text { minutos, luego del } \\
\text { efecto anestésico alcanzado para preparar el tendón } \\
\text { para la manipulación de Mills }\end{array}$ & $\begin{array}{l}\text { Seguimiento: No posee } \\
\text { - Dolor (EVA) } \\
\text { - TEFS (Funcionalidad) }\end{array}$ \\
\hline
\end{tabular}

MDA: método de aleatorización; EL: epicondilalgia lateral; EVA: escala visual análoga; SD: desviación estándar; X: promedio; DASH: cuestionario de discapacidad de hombro, brazo y mano; GQL: instrumento de calidad de vida; PFFQ: cuestionario de función libre de dolor modificado; EMM: máxima elongación muscular; CVM: contracción máxima voluntaria; PRTEE: evaluación del paciente con mejor puntuación en EL; PRTEEQ: cuestionario de evaluación del paciente con mejor puntuación; PRFEQ: cuestionario de evaluación relacionado con el antebrazo del paciente; GRIPPIT: escala de evaluación de fuerza y resistencia; TEFS: escala de función de codo de tenista; MEPS: escala de performance de mayo de codo de tenista.

\section{DISCUSIÓN}

Se intentó determinar si existe evidencia científica que avale la efectividad clínica del ejercicio terapéutico sólo o dentro de un programa de tratamiento en comparación con otra intervención fisioterapéutica en pacientes adultos con epicondilalgia lateral. Para lograrlo se realizó una síntesis de la evidencia disponible para responder la interrogante planteada precedentemente. En un comienzo debido a la amplia variedad y compleja clasificación de todos los ejercicios terapéuticos se establecieron criterios de inclusión y exclusión para acotar la variabilidad de ejercicios propuestos. De acuerdo a esto según la clasificación de Kisner (60) adicionado a los criterios ya establecidos se propusieron los ejercicios terapéuticos más comunes como: ejercicios concéntricos, ejercicios excéntricos, ejercicios de resistencia, ejercicios sensoriomotrices, ejercicios de estiramientos, ejercicio isocinético y programa de ejercicio en casa. Según lo planteado, nuestra búsqueda arrojó 36 artículos seleccionados que no estaban en condiciones de agruparse en base a un estimador puntual, debido a la heterogeneidad de la naturalidad de las intervenciones de esta revisión sistemática sobre ejercicio terapéutico, se hace difícil la extracción de datos, entendiendo así que para llevar a cabo un meta-análisis se debe evaluar además la homogeneidad estadística, que es menos plausible de realizar debido que no se cumple el primer criterio propuesto. Por este motivo se utilizó un método cualitativo con el uso de niveles de evidencia para la síntesis de los datos, situación que es difícil para el análisis final ya que carece de un poder estadístico que proporcione pruebas concluyentes para la extrapolación clínica. En cuanto a la evaluación del riesgo de sesgo de los artículos incluidos (riesgo de sobreestimar o subestimar los resultados) realizado por los autores de la presente revisión se establecieron criterios para designar bajo riesgo sesgo, alto riesgo sesgo y riesgo de sesgo incierto. En el primer punto de la evalua- 
ción que corresponde a la generación de la secuencia aleatoria se consideró bajo riesgo de sesgo cuando: se utiliza un generador de secuencia aleatoria, sorteo, utilización de una moneda, lanzamiento de dados. Alto riesgo de sesgo cuando: Secuencia generada por fecha de nacimiento, según días de admisión, secuencia generada por juicio clínico. Riesgo de sesgo incierto cuando: si el método de secuencia no se describe o falta información sobre el proceso. En el punto de cegamiento de participantes, terapeutas y evaluador, se decidió realizar un consenso y juicio sobre la evaluación de este punto. Debido a la naturaleza de la intervención en este caso el ejercicio, resulta difícil cegar al paciente o al terapeuta, ya que siempre se conoce la terapia a realizar. Por lo tanto se consideró Bajo riesgo de sesgo en este punto para todos los artículos. Sin embargo para el cegamiento de los evaluadores se puede castigar categóricamente si no cumple este punto. Es considerado Bajo riesgo de sesgo cuando: Si se reporta en el estudio. Alto riesgo de sesgo: Si no lo reporta en el estudio. Riesgo de sesgo incierto: Cuando el ocultamiento no se describe o falta información sobre el proceso. De todos los estudios seleccionados ninguno tiene inscrito el protocolo de investigación en algún sistema de registro existente, sin embargo, presentaban los datos reportados para cada estudio. De acuerdo a esto, tienen bajo riesgo de sesgo; Bisset (23), Bisset (45), Blanchette (17), Chung (22), Hernandez (21), Kochar (27), Nagrale (31), Peterson (15), Pienimaki (7), Radpasand (16), Smidt (43), Stasinopoulos (72), Stasinopoulos (83), Stergioulas (14), Struijs (37), Struijs (19), Tyler (82), Verhaar (39), Viswas (11), con moderado riesgo de sesgo; Ajimsha (18), Bhardwaj (32), Coff (9), Croisier (73), Dreschler (29), Manias (44), Martinez-Silvestrini (74), Ho (12), Oken (8), Park (84), Soderberg (20), Stasinopoulos (38) y con alto riesgo de sesgo; Luginbuhl (75), Newcomer (40), Nilsson (25), Pienimaki (61), Svernlov (33). Existe una RS publicada por Raman (92) que indica que los ejercicios de resistencia pueden reducir el dolor, aumentar la fuerza, y la función en EL, sin embargo si existe una intervención variable es el ejercicio, por lo cual, resulta complejo establecer una dosis óptima y específica para tal condición, lo que hace más difícil la aplicabilidad de los resultados. En cuanto a la comparación del ejercicio sólo versus cualquier otra intervención sola, dentro de los 4 artículos $(15,18,33,61)$ agrupados, en los estudios de Peterson (15) y Ajimsha (18) establecen diferencias significativas a favor del grupo de ejercicios en las medidas de resultados de dolor y funcionalidad a corto plazo. En el estudio de Pienimaki (61) muestra diferencias significativas en la medida de resultado de dolor en el grupo de ejercicio a largo plazo. En el estudio de Svernlov (33) mostró diferencias significativas en la medida de resultado de dolor y grip libre de dolor para ambos grupos a mediano plazo. Cabe consignar que ambos grupos eran de distinto tipo de ejercicio. En cuanto a la comparación de un programa de tratamiento con ejercicio terapéutico versus una intervención sola, dentro de los 17 artículos seleccionados. Los estudios de Ho (12), Pienimaki (7), Bisset (23), Soderberg (20), Smidt (46), Struijs (19), Verhaar (41), Bhardwaj (34) establecen diferencias significativas a favor del programa de tratamiento con ejercicio versus una intervención sola en las medidas de resultados de dolor a corto plazo. Cabe consignar que en el estudio Bisset (23), existe una disminución del dolor a corto plazo atribuida a la inyección de corticoides, pero a largo plazo era favorable al grupo de ejercicios. En cuanto al grip libre de dolor existen diferencias significativas a corto plazo $(7,12,19,23,41,46)$. En los estudios de Struijs (39), Croisier (76) establecen diferencias significativas a favor de la intervenciones que no poseían ejercicios (39) y a la intervención sola (76), en las medidas de resultados de dolor existe una disminución a corto plazo. En cuanto al grip libre de dolor existe diferencias significativas a corto plazo (39).Los estudios restantes de la comparación no establecen diferencias significativas en las medidas de resultados establecidas. En cuanto a la comparación de ejercicio terapéutico dentro de un programa de tratamiento versus otro programa de tratamiento, dentro de los 15 artículos seleccionados, los estudios de Chung (22), Park (84), Viswas (11), establecieron diferencias significativas a favor del programa de tratamiento con ejercicio terapéutico en comparación con otro programa de tratamiento en las medidas de resultados de dolor a corto plazo. Los estudios de Stergioulas (13), Kochar (28), Radpasand (16), Stasinopoulos (86) establecieron diferencias significativas a favor del programa de tratamiento con ejercicio terapéutico en comparación con otro programa de tratamiento en las medidas de resultados de dolor a mediano plazo. Los estudios de Hernández (21), Nilsson (25), Park (84), Radpasand (16), Stasinopoulos (86), Tyler (85), Viswas (11), Stasinopoulos (86) establecieron diferencias significativas a favor del programa de tratamiento con ejercicio terapéutico en comparación con otro programa de tratamiento en las medidas de resultados de la funcionalidad a corto, mediano y largo plazo. En cuanto al grip libre de dolor, los estudios de Stasinopoulos (86), Stergioulas (13), Tyler (85), Viswas (11) establecieron diferencias significativas a favor del programa de tratamiento con ejercicio terapéutico en comparación con otro programa de tratamiento en la medida de resultado grip libre de dolor a corto y mediano plazo.

Cabe señalar que las múltiples diferencias en los tipos y dosis de ejercicios deben ser consideraciones a evaluar cuando se interpreten los resultados de los diferentes estudios y se intenten establecer conclusiones acerca de la efectividad clínica de las intervenciones terapéuticas estudiadas.

\section{CONCLUSIÓN}

A pesar de la aparente homogeneidad clínica que presentaban algunos de los artículos seleccionados por nuestra 
RS, era sumamente variable la dosis y el tipo de ejercicio, lo cual hace difícil realizar las comparaciones propuestas anteriormente, estas consignaciones no permitieron el agrupamiento estadístico de los datos en base a un estimador puntual, aún así igual se consideró pertinente realizar un agrupamiento cualitativo o descriptivo de los resultados en las intervenciones clínicamente similares. Para la agrupación de ejercicio sólo comparado con una intervención sola existe moderada evidencia que el ejercicio terapéutico disminuye el dolor, mejora la funcionalidad y aumenta la fuerza libre de dolor en corto, mediano y largo plazo. Para la agrupación de ejercicio dentro de un programa de tratamiento comparado con una intervención sola existe sólida evidencia que el ejercicio terapéutico disminuye el dolor, y aumenta la fuerza libre de dolor a corto plazo. Para la agrupación de ejercicio terapéutico dentro de un programa de tratamiento comparado con otro programa de tratamiento (Laser, Tens, US) existe sólida evidencia que el ejercicio terapéutico disminuye el dolor, aumenta la fuerza y mejora la funcionalidad a corto, mediano y largo plazo. Cabe consignar que las conclusiones de nuestro estudio se realizaron sobre la base de los artículos localizados por nuestra estrategia de búsqueda y seleccionados por nuestros criterios de elegibilidad; aún así, siempre se debe tomar en cuenta la probabilidad de que haya estudios que por problemas de indexación o límites de búsqueda hayan quedado fuera de esta revisión. Por último se agradece a los autores que de forma desinteresada proporcionaron información adicional para realizar esta presente RS.

\section{CORRESPONDENCIA:}

Felipe Araya

e-mail: fandres.kine@gmail.com

\section{BIBLIOGRAFÍA}

1. Maffulli N, Kahn KM, Puddu G. Overuse tendon conditions: Time to change a confusing terminology. Arthr 1998 Nov; 14(8):840-3.

2. Vicenzino B, Wright A. Lateral epicondylalgia. I. Epidemiology, pathophysiology, etiology and natural history. Phys Ther Rev 1996 Sep; 1:23-34.

3. Waugh EJ, Jaglal SB, Davis AM, Tomlinson G, Verrier MC. Factors associated with prognosis of lateral epicondylitis after 8 weeks of physical therapy. Arch Phys Med Rehabil 2004; 85(2):308-18.

4. Haker E. Lateral epicondylalgia: diagnosis, treatment and evaluation. Crit Rev Phys Rehabil Med 1993; 5:129-54.

5. Assendelft W, Green S, Buchbinder R, Struijs P, Smidt N. Tennis elbow. BMJ 2003 Aug; 327(7410):329.

6. Vasseljen O. Low-level laser versus traditional physiotherapy in the treatment of tennis elbow. Physio 1992 May; 78(5):329-4.

7. Pienimaki T, Tarvainen T, Siira P, Vanharanta H. Progressive strengthening and stretching exercises and ultrasound for chronic lateral epicondylitis. Phys 1996 Sep; 82(9):522-30.

8. Oken O, Kahraman Y, Ayhan F, Canpolat S, Yorgancioglu ZR, Oken OF. The Short-term Efficacy of Laser, Brace, and Ultrasound Treatment in Lateral Epicondylitis: A Prospective, Randomized, Controlled Trial. J Hand Ther 2008 JanMar; 21(1):63-7.

9. Coff L, Massy-Westropp N, Caragianis S. Randomized controlled trial of a new electrical modality (inter X) and soft tissue massage, stretching, ultrasound and exercise for treating lateral epicondylitis. J Hand Ther 2009 Jun; 14(2):46-52.

10. Cyriax JH. The pathology and treatment of tennis elbow. J Bone Joint Surg 1936 Oct; 18(4):921-40.

11. Viswas R, Ramachandran R, Korde Anantkumar P. Comparison of effectiveness of supervised exercise program and Cyriax physiotherapy in patients with tennis elbow (lateral epicondylitis): a randomized clinical trial. Scientific World Journal. 2012 May; 2012: 645-939.

12. Ho L, Kwong W, Cheing G. Effectiveness of Microcurrent Therapy in the Management of Lateral Epicondylitis: A Pilot Study. Hong Kong Physiother J 2007 Nov; 25(1):14-20.

13. Allander E. Prevalence, incidence and remission rates of some common rheumatic diseases and syndromes. Scand J Rheumatol 1974; 3(3):145-53.

14. Stergioulas A. Effects of Low-Level Laser and Plyometric Exercises in the Treatment of Lateral Epicondylitis. Photomed Laser Surg. 2007 Jun; 25(3):205-13.

15. Peterson M, Butler S, Eriksson M, Svardsudd K. A randomized controlled trial of exercise versus wait-list in chronic tennis elbow (lateral epicondylosis). J Med Sci 2011 Nov; 116(4): 269-79.

16. Radpasand M, Owens E. Combined multimodal therapies for chronic tennis elbow: pilot study to test protocols for a randomized clinical trial. J Manipulative Physiol Ther 2009 Sep; 32(7):571-85.

17. Blanchette MA, Normand MC. Augmented soft tissue mobilization vs natural history in the treatment of lateral epicondylitis: a pilot study. J Manipulative Physiol Ther 2011 Feb; 34(2):123-30.

18. Ajimsha MS, Chithra S, Thulasyammal RP. Effectiveness of Myofascial Release in the Management of Lateral Epicondylitis in Computer Professionals. Arch Phys Med Rehabil 2012 Apr; 93(4):604-9.

19. Struijs P, Kerkhoffs G, Assendelft W, Van Dijk C. Consevative treatment of lateral epicondylitis: brace versus physical therapy or a combination of both- a randomized clinical trial. Am J Sports Med 2004 Mar; 32(2):462-9.

20. Soderberg J, Grooten W, Ang B. Effects of eccentric training on hand strength in subjects with lateral epicondylalgia: a randomized-controlled trial. Scand J Med Sci Sports 2011.

21. Hernández D, Berjillos A, de la Corte H, Díaz F, Tena C, Moraleda S, et al. Estudio prospectivo randomizado de comparación de diferentes técnicas de electroterapia en tendinitis de inserción en codo. Patología del aparato locomotor 2006; 4(2):131-8.

22. Chung B, Wiley P. Effectiveness of Extracorporeal Shock Wave Therapy in the Treatment of Previously Untreated Lateral Epicondylitis: A Randomized Controlled Trial. Am J Sports Med 2004 oct-Nov; 32(7):1660-7.

23. Bisset L, Beller E, Jull G, Brooks P, Darnell R, Vicenzino B. Mobilisation with movement and exercise, corticosteroid injection, or wait and see for tennis elbow: randomised trial. BMJ 2006 Nov; 333(7575):939-41. 
24. Vicenzino B. Lateral epicondilalgia: a musculoskeletal physiotherapy perspective. Man Ther 2003 May; 8(2):66-79.

25. Nilsson P, Thom E, Baigi A, Marklund B, Mansson J. A prospective pilot study of a multidisciplinary home training programme for lateral epicondylitis. Musculoskeletal Care 2007 Mar; 5(1):36-50.

26. Verhaar J. Tennis elbow: anatomical, epidemiological and therapeutic aspects. Int Orthop 1994 Oct; 18(5):263-7.

27. Kochar M, Dogra A. Effectiveness of a Specific Physiotherapy Regimen on Patients with Tennis Elbow: Clinical study. Phys 2002; 88(6):333-41.

28. Hudak PL, Cole DC, Haines AT. Understanding prognosis to improve rehabilitation: the example of lateral elbow pain. Arch Phys Med Rehabil. 1996 Jun; 77(6):586-93

29. Drechsler W, Knarr J, Snyder-Mackler L. A Comparison of Two Treatment Regimens for Lateral Epicondylitis: A Randomized Trial of Clinical Interventions. J Sport Rehabil 1997 Aug; 6(3):226-34.

30. Abate M, Silbernagel KG, Siljeholm C, Di Iorio A, De Amicis $\mathrm{D}$, Salini V, et al. Pathogenesis of tendinopathies: inflammation or degeneration?. Arthritis Res Ther 2009 jun; 11(3):235.

31. Nagrale A, Herd C, Ganvir S, Ramteke G. Cyriax. Physiotherapy versus Phonophoresis with supervised exercise in subjects with Lateral epicondylalgia: A Randomized Clinical trial. J Man Manip Ther 2009; 17(3):171-8.

32. Bhardwaj P, Dhawan A. The relative efficacy of mobilization with movement versus Cyriax physiotherapy in the treatment of lateral epicondylitis. Indian Journal of Physiotherapy and Occupational Therapy 2011; 5(1):142-6.

33. Svernlov B, Adolfsson L. Non-operative treatment regime including eccentric training for lateral humeral epicondylalgia. Scand J Med Sci Sports 2001 Dec; 11(6):328-34.

34. Stasinopoulos D, Stasinopoulos I. Comparison of effects of Cyriax physiotherapy, a supervised exercise programme and polarized polychromatic non-coherent light (Bioptron light) for the treatment of lateral epicondylitis. Clin Rehabil 2006 Jan; 20(1):12-23.

35. Birch HL, Wilson AM, Goodship AE. The effect of exerciseinduced localised hyperthermia on tendon cell survival. J Exp Biol 1997 Jun; 200(11):1703-8.

36. Wren TA, Lindsey DP, Beaupre GS, Carter DR. Effects of creep and cyclic loading on the mechanical properties and Failure of Human Achilles Tendons. ABME 2003 Jun;31(6):710-17.

37. Struijs PA, Damen PJ, Bakker EW, Blankevoort L, Assendelft WJ, Van Dijk CN. Manipulation of the Wrist for Management of Lateral Epicondylitis: A Randomized Pilot Study. Phys Ther 2003 Jul; 83(7):608-16.

38. Stasinopoulos D, Stasinopoulos I, Pantelis M, Stasinopoulos K. Comparing the Effects of Exercise Program and LowLevel Laser Therapy with Exercise Program and Polarized Polychromatic Non-coherent Light (Bioptron Light) on the Treatment of Lateral Elbow Tendinopathy. Photomed Laser Surg 2009 Jun; 27(3):513-20.

39. Verhaar J, Walenkamp G, Van Mameren H, Kester A, Van der Linden A. Local corticosteroid injection versus Cyriaxtype physiotherapy for tennis elbow. J Bone Joint Surg Br 1996 Jan; 78(1):128-32.

40. Newcomer KL, Laskowski ER, Idank DM, McLean TJ, Egan KS. Corticosteroid Injection in Early Treatment of Lateral Epicondylitis. Clin J Sport Med 2001 Oct; 11(4):214-22.

41. Ashe MC, McCauley T, Khan KM. Tendinopathies in the upper extremity: a paradigm shift. J Hand Ther 2004 JulSep; 17(3):329-34.
42. Wang JH, Iosifidis MI, Fu FH. Biomechanical basis for tendinopathy. Clin Orthop Relat Res 2006 Feb; 443:320-32.

43. Smidt N, Van der Windt DA, Assendelft WJ, Devillé WL, Korthals-de Bos IB, Bouter LM. Corticosteroid injections, physiotherapy, or a wait-and-see policy for lateral epicondylitis: a randomised controlled trial. Lancet 2002 Feb; 359(9307):657-62.

44. Manias P, Stasinopoulos D. A controlled clinical pilot trial to study the effectiveness of ice as a supplement to the exercise programme for the management of lateral elbow tendinopathy. Br J Sports Med 2006 Jan; 40(1):81-85.

45. Bisset LM, Coppieters MW, Vicenzino B. Sensorimotor Deficits Remain despite Resolution of Symptoms using conservative treatment in patients with tennis elbow: A randomized controlled trial. Arch Phys Med Rehabil 2009 Jan; 90(1):1-8

46. Pienimaki TT, Kauranen K, Vanharanta H. Bilaterally decreased motor performance of arms in patients with chronic tennis elbow. Arch Phys Med Rehabil 1997 Oct; 78(10):1092-5.

47. Bisset LM, Russell T, Bradley S, Ha B, Vicenzino BT. Bilateral sensorimotor abnormalities in unilateral lateral epicondylalgia. Arc Phys Med Rehabil 2006 Apr; 87(4):490-5.

48. Lephart S, Fu F. Proprioception and Neuromuscular Control in Joint Stability. Human Kinetics; 2000; p 17-24.

49. Riemann B, Lephart S. The sensorimotor system, part I: The physiological basis of functional joint stability. J Athl Train 2002 Jan-Mar; 37(1):71-9.

50. Cowan SM, Bennell KL, Hodges PW, Crossley KM, McConnell J. Delayed onset of electromyographic activity of vastus medialis obliquus relative to vastus lateralis in subjects with patellofemoral pain syndrome. Arch Phys Med Rehabil 2001 Feb; 82(2):183-9.

51. Hodges PW, Moseley GL. Pain and motor control of the lumbopelvic region: effect and possible mechanisms. J Electromyogr Kinesiol 2003 Aug; 13(4):361-70.

52. Hodges PW, Richardson CA. Inefficient muscular stabilization of the lumbar spine associated with low back pain: a motor control evaluation of transversus abdominis. Spine 1996 Nov; 21(22):2640-50.

53. Barden JM, Balyk R, Raso VJ, Moreau M,Bagnall K. Dynamic upper limb propioception in multidirectional shoulder instability. Clin Orthop 2004;(420):181-89.

54. Myers JB, Wassinger CA, Lephart SM. Sensorimotor contribution to shoulder stability: effect of injury and rehabilitation. Man Ther 2006 Aug; 11(3):197-201.

55. Smidt N, Van der Windt DA, Assendenlft WJ, Devillé WL, Korthals-de Bos IB, Bouter LM. Corticosteroid injections, physiotherapy, or a wait and see policy for lateral epicondilitis: a randomized controlled trail. J Phys Ther 2002; 359(9307):657-62.

56. Hay E, Peterson S, Lewis M, Hosie G, Croft P. Pragmatic randomized controlled trial of local corticosteroid injects and naproxen for treatment of lateral epicondilitis of elbow in primary care. Br Med J.1999 Oct; 319(7215); 964-8.

57. Kisner C, Colby L.A. Therapeutic Exercise: Foundations and Techniques. 5 ed. F.A. Davis Company, 2007

58. Wright A, Vicenzino B. Lateral epicondylalgia II: therapeutic management. Phys Ther Rev 1997 Mar; 2(1):39-48.

59. Pienimaki T. Conservative treatment and rehabilitation of tennis elbow: a review article. Crit Rev Phys Rehabil Med 2000; 12:213-28.

60. Selvier T, Wilson J. Methods utilized in treating lateral epicondylitis. Phys Ther Rev 2000 Jun; 5(2):117-24. 
61. Pienimaki T, Karinen P, Kemila T, Koivukangas P, Vanharanta H. Long-term follow-up of conservatively treated chronic tennis elbow patients. A prospective and retrospective analysis. Scand J Rehabil Med. 1998 Sep; 30(3):159-66.

62. Noteboom T, Cruver R, Keller A, Keller J, Kellogg B, Nitz A. Tennis elbow: a review. J Orthop Sports Phys Ther 1994 Jun; 19(6):357-66.

63. Khan K, Cook J, Taunton J, Bonar F. Overuse tendinosis, not tendinitis: a new paradigm for a difficult clinical problem. Phys Sports med 2000 May; 28(5):38-48.

64. Selvier T, Wilson J. Treating lateral epicondylitis. Sports Med 1999 Nov; 28(5):375-80.

65. Ohberg L, Lorentzon R, Alfredson H, Maffulli N. Eccentric training in patients with Achilles tendinosis: normalized tendon structure and decreased thickness at follow up. Br J Sports Med 2004 Feb; 38(1):8-11.

66. Ohberg L, Lorentzon R, Alfredson H. Neovascularisation in Achilles tendons with painful tendinosis but not in normal tendons: an ultrasonographic investigation. Knee Surg Sports Traumatol Arthr 2001 Jul; 9(4):233-8.

67. Koltyn KF, Arbogast RW. Perception of pain after resistance exercise. Br J Sports Med 1998 March; 32(1):20-4.

68. Kosek E, Lundberg L. Segmental and plurisegmental modulation of pressure pain thresholds during static muscle contractions in healthy individuals. Eur J Pain 2003; 7(3):251-8.

69. Marqueste T, Decherchi P, Messan F, Kipson N, Grelot L, Jammes Y. Eccentric exercise alters muscle sensory motor control through the release of inflammatory mediators. Brain Res 2004 Oct; 1023(2):222-30.

70. Staud R, Robinson ME, Price DD. Isometric exercise has opposite effects on central pain mechanisms in fibromyalgia patients compared to normal controls. Pain 2005 Nov; 118(1-2):176-84.

71. Gibson W, Arendt-Nielsen L, Graven-Nielsen T. Referred pain and hyperalgesia in human tendon and muscle belly tissue. Pain 2006 Jan; 120(1-2):113-23.

72. Stasinopoulos D, Stasinopoulos K, Johnson MI. An exercise programme for the management of lateral elbow tendinopathy. Br J Sports Med 2006; 39:944-7.

73. Croisier JL, Foidart-Dessalle M, Tinant F, Crielaard JM, Forthomme B. An isokinetic eccentric programme for the management of chronic lateral epicondylar tendinopathy. $\mathrm{Br}$ J Sports Med 2007 April; 41(4): 269-75.

74. Martínez-Silvestrini JA, Newcomer KL, Gay RE, Schaefer MP, Kortebein P, Arendt KW. Chronic lateral epicondylitis: comparative effectiveness of a home exercise program including stretching alone versus stretching supplemented with eccentric or concentric strengthening. J Hand Ther 2005 Oct-Dec; 18(4):411-9.

75. Luginbühl R, Brunner F, Schneeberger A. No effect of forearm band and extensor strengthening exercises for the treatment of tennis elbow: a prospective randomized study. Chir Organi Mov 2008 Jan; 91(1):35-40.
76. Kraushaar B, Nirschl R. Current concepts review: Tendinosis of the elbow (tennis elbow). Clinical features and findings of histological, immunohistochemical and electron microscopy studies. J Bone Joint Surg 1999 Feb; 81(2): 259-78.

77. Hawary R, Stanish W, Curwin S. Rehabilitation of tendon injuries in sport. Sports Med 1997 Nov; 24(5):347-58.

78. Stanish W, Curwin S, Mandell S. Tendinitis: its etiology and treatment. Br J Sports Med 2001; 35:139.

79. Stanish WD, Rubinovich RM, Curwin S. Eccentric exercise in chronic tendinitis. Clin Orthop 1986 Jul;(208):65-8.

80. Fyfe I, Stanish WD. The use of eccentric training and stretching in the treatment and prevention of tendon injuries. Clinic Sports Med 1992 Jul; 11(3):601-24.

81. Hawary R, Stanish WD, Curwin SL. Rehabilitation of tendon injuries in sport. Sports Med 1997 Nov; 24(5):347-58.

82. Tyler TF, Thomas GC, Nicholas SJ, McHugh MP. Addition of isolated wrist extensor eccentric exercise to standard treatment for chronic lateral epicondylosis: A prospective randomized trial. J Shoulder Elbow Surg 2010 Sep; 19(6):917-22.

83. Stasinopoulos D, Stasinopoulos I, Pantelis M, Stasinopoulos $\mathrm{K}$. Comparison of effects of a home exercise programme and a supervised exercise programme for the management of lateral elbow tendinopathy. Br J Sports Med 2010 Jun; 44(8):579-83.

84. Park JY, Park HK, Choi JH, Moon ES, Kim BS, Kim WS, et al. Prospective Evaluation of the Effectiveness of a Home-Based Program of Isometric Strengthening Exercises: 12-Month Follow-up. Clin Orthop Surg 2010 Sep; 2(3):173-8.

85. Alter M. Sport stretch. 2 ed. Champaign, IL: Human Kinetics, 1998.

86. Prentice W. Rehabilitation techniques in sports medicine. 5 ed. Boston: McGraw-Hill, 2010

87. Shrier I, Gossal K. Myths and truths of stretching. Phys Sports med 2000 Aug; 28(8):57-63.

88. Taylor DC, Dalton JD Jr, Seaber AV, Garrett WE Jr. Viscoelastic properties of muscle-tendon units: the biomechanical effects of stretching. Am J Sports Med 1990 MayJun;18(3):300-9.

89. Herling J. It's time to add strength training to our fitness programs. J Phys Educ Programme 1981; 79:17.

90. Bandy WD, Irion JM, Briggler M. The effect of time and frequency of static stretching on flexibility of the hamstring muscles. Phys Ther 1997 Oct; 77(10):1090-6.

91. Van Tulder M, Furlan A, Bombardier C, Bouter L. Editorial board of the Cochrane Collaboration back review group. Updated method guidelines for systematic reviews in the Cochrane Collaboration back review group. Spine. 2003;28:1290-9.

92. Raman J, MacDermid JC, Grewal R. Effectiveness of Different Methods of Resistance Exercises in Lateral Epicondylosis: A Systematic Review. J Hand Ther 2012 Jan; 25(1): $5-26$ 